ARTICLE

\title{
The P-type ATPase transporter ATP7A promotes angiogenesis by limiting autophagic degradation of VEGFR2
}

\author{
Dipankar Ash (1) 1,10, Varadarajan Sudhahar 1,2,10, Seock-Won Youn 1,3, Mustafa Nazir Okur ${ }^{4}$, Archita Das ${ }^{1}$, \\ John P. O'Bryan ${ }^{5,6}$, Maggie McMenamin ${ }^{1,2}$, Yali Hou ${ }^{1,2}$, Jack H. Kaplan7, Tohru Fukai (1) 1,8,2,11凶 \& \\ Masuko Ushio-Fukai (1, $1,9,11 \bowtie$
}

VEGFR2 (KDR/FIk1) signaling in endothelial cells (ECs) plays a central role in angiogenesis. The P-type ATPase transporter ATP7A regulates copper homeostasis, and its role in VEGFR2 signaling and angiogenesis is entirely unknown. Here, we describe the unexpected crosstalk between the Copper transporter ATP7A, autophagy, and VEGFR2 degradation. The functional significance of this Copper transporter was demonstrated by the finding that inducible EC-specific ATP7A deficient mice or ATP7A-dysfunctional ATP7Amut mice showed impaired post-ischemic neovascularization. In ECs, loss of ATP7A inhibited VEGFinduced VEGFR2 signaling and angiogenic responses, in part by promoting ligand-induced VEGFR2 protein degradation. Mechanistically, VEGF stimulated ATP7A translocation from the trans-Golgi network to the plasma membrane where it bound to VEGFR2, which prevented autophagy-mediated lysosomal VEGFR2 degradation by inhibiting autophagic cargo/ adapter p62/SQSTM1 binding to ubiquitinated VEGFR2. Enhanced autophagy flux due to ATP7A dysfunction in vivo was confirmed by autophagy reporter CAG-ATP7Amut -RFPEGFP-LC3 transgenic mice. In summary, our study uncovers a novel function of ATP7A to limit autophagy-mediated degradation of VEGFR2, thereby promoting VEGFR2 signaling and angiogenesis, which restores perfusion recovery and neovascularization. Thus, endothelial ATP7A is identified as a potential therapeutic target for treatment of ischemic cardiovascular diseases.

\footnotetext{
${ }^{1}$ Vascular Biology Center, Medical College of Georgia at Augusta University, Augusta, GA, USA. ${ }^{2}$ Charlie Norwood Veterans Affairs Medical Center, Augusta, GA, USA. ${ }^{3}$ Department of Physiology and Biophysics, University of Illinois College of Medicine, Chicago, IL, USA. ${ }^{4}$ Laboratory of Molecular Gerontology, National Institute on Aging, National Institutes of Health, Baltimore, MD, USA. ${ }^{5}$ Department of Cell and Molecular Pharmacology and Experimental Therapeutics, Hollings Cancer Center, Medical University of South Carolina, Charleston, SC, USA. ${ }^{6}$ Ralph H. Johnson VA Medical Center, Charleston, SC, USA. ${ }^{7}$ Department of Biochemistry and Molecular Genetics, University of Illinois College of Medicine, Chicago, IL, USA. ${ }^{8}$ Departments of Pharmacology and Toxicology, Medical College of Georgia at Augusta University, Augusta, GA, USA. ${ }^{9}$ Department of Medicine (Cardiology), Medical College of Georgia at Augusta University, Augusta, GA, USA. ${ }^{10}$ These authors contributed equally: Dipankar Ash, Varadarajan Sudhahar. ${ }^{11}$ These authors jointly supervised this work: Tohru Fukai, Masuko Ushio-Fukai. ${ }^{凶}$ email: tfukai@augusta.edu; mfukai@augusta.edu
} 
A ngiogenesis, the process of new vessel formation from pre-existing vessels, plays important role in wound repair and restoring perfusion recovery and neovascularization in ischemic heart disease and peripheral vascular disease ${ }^{1}$. Vascular endothelial growth factor (VEGF), a key angiogenic growth factor, stimulates migration, proliferation, and capillary tube formation of endothelial cells (ECs) primarily through VEGF receptor type2 (VEGFR2), which promotes neovascularization and vascular regeneration ${ }^{2}$. VEGFR2 is a classic tyrosine kinase receptor, having an intracellular kinase domain that is activated upon ligand binding and dimerization, resulting in autophosphorylation, and activation of intracellular signaling, via MAPK and PI3K/Akt. Like other receptor tyrosine kinases, VEGFR2 was thought to signal from the cell plasma membrane after ligandinduced dimerization and activation ${ }^{3}$. However, a number of recent studies have challenged this notion by showing the importance of endocytosis and trafficking of VEGFR2 in regulating its signaling ${ }^{2}$. Upon VEGF stimulation, activated/dimerized VEGFR2 at the cell surface is internalized to early endosomes for activating signaling, but some fraction of the receptor is ubiquitinated and sorted to the lysosome for degradation, the remaining is recycled to the plasma membrane ${ }^{4}$. However, the molecular mechanisms that regulate VEGFR2 signaling via endocytosis and trafficking remain elusive.

The P-type ATPase transporter ATP7A is a key regulator of secretory $\mathrm{Cu}$ enzymes and of intracellular $\mathrm{Cu}$ levels ${ }^{5,6}$. Under basal conditions, ATP7A localizes at the trans-Golgi network (TGN) where it transports $\mathrm{Cu}$ to the secretary $\mathrm{Cu}$ enzymes, such as extracellular superoxide dismutase (SOD3) or the proenzyme of lysyl oxidase (Pro-LOX) required for LOX activation ${ }^{5,6}$, which promotes tumorigenesis and metastasis ${ }^{7}$. It is also partially involved in VEGF or ischemia-induced angiogenesis in $\mathrm{ECs}^{8,9}$. In pathological conditions in which cellular $\mathrm{Cu}$ is elevated, ATP7A translocates from TGN to the plasma membrane to export the excess $\mathrm{Cu}$. It has been shown that relocalization of ATP7A from the TGN is triggered not only by increased cytoplasmic $\mathrm{Cu}$ but also by non-metal stimulants such as insulin, NMDA, PDGF, and hypoxia ${ }^{10}$. The biological significance of ATP7A in vivo is underscored by Menkes disease which is caused by a loss-offunction X-linked mutation of ATP7A ${ }^{11}$. Of note, global ATP7A knockout mice are embryonic lethal due to vascular defects ${ }^{12}$. Mice carrying the X-linked blotchy ATP7A mutation (ATP7A ${ }^{\text {mut }}$ mice) have a splice site mutation introducing a new stop codon at amino acid residue 794 with reduced $\mathrm{Cu}$ transport function and ATP7A expression. These mutant mice survive more than 6 months of age $e^{5,13-15}$ and are well characterized to enable the study of the in vivo function of ATP7A in adult animals. Using ATP7A $^{\text {mut }}$ mice, we reported that vascular ATP7A protects against endothelial dysfunction in hypertensive ${ }^{14}$ and in diabetic mice ${ }^{16,17}$. We also showed that ATP7A protein expression was markedly downregulated in diabetic vessels, which led to impaired endothelium-dependent vasorelaxation ${ }^{16,17}$. However, the role of endothelial ATP7A in VEGF-induced angiogenesis in ECs and postnatal angiogenesis in vivo has not been explored.

Autophagy is an evolutionarily conserved lysosomal degradation pathway mediating the clearance of damaged organelle and dysfunctional proteins within autophagosomes which fuse with acidic lysosomes ${ }^{18}$. The cargo has several components, including a selective autophagy adapter/cargo p62/Sequestosome 1 (SQSTM1) to detect and aggregate polyubiquitin proteins and bind to the integral autophagosome component LC3 for autophagosome generation ${ }^{19}$. It is degraded by lysosomal proteases to provide essential elements for maintaining cell metabolism ${ }^{20}$. However, the role of autophagy in angiogenesis is complex. For example, ATG5-mediated autophagy induced by nutrient deprivation is required for angiogenesis ${ }^{21}$, while autophagy induced by glycolytic intermediate impaired angiogenesis 22 . Furthermore, there is a connection between autophagy, lysosomes, and the $\mathrm{Cu}$ transport proteins involved in regulating $\mathrm{Cu}$ metabolism. It is known that late endosomes/lysosomes import $\mathrm{Cu}$ via the ATP7A homolog, ATP7B, which moves to the lysosome from the TGN in response to $\mathrm{Cu}$ in hepatic cells ${ }^{23}$; induction of autophagy protects ATP7B deficient hepatocytes from excess $\mathrm{Cu}$-mediated toxicity ${ }^{24}$; $\mathrm{Cu}$ accumulation in senescent mouse fibroblasts is due to the impaired interplay between ATP7A and the autophagiclysosomal pathway ${ }^{25}$. However, the mechanistic linkages between ATP7A and autophagy in VEGFR2 signaling in ECs and neovascularization in vivo have not been characterized.

In this study, using inducible endothelial-restricted ATP7A knockout (iEC-ATP7A KO) mice obtained by crossing ATP7A $\mathrm{A}^{\mathrm{fl} / \mathrm{fl}}$ with tamoxifen-inducible iCdh5-CreERT2 mice ${ }^{26}$ or using ATP7A dysfunctional ATP7A ${ }^{\text {mut }}$ mice, we provide evidence that these ATP7A dysfunctional mice exhibit reduced ischemiainduced angiogenesis. We found an endothelial ATP7Aautophagy linkage, which is important in the regulation of VEGFR2 signaling. Loss of ATP7A in ECs promoted autophagosome formation and autophagic cargo/adapter p62/SQSTM1 binding to ubiquitinated VEGFR2, thereby accelerating VEGFR2 targeting to autophagolysosomes for degradation (in a $\mathrm{Cu}$ independent manner), which in turn inhibits VEGF-induced angiogenesis. Thus, endothelial ATP7A functions to promote VEGFR2 signaling and angiogenesis by limiting autophagic degradation of VEGFR2, in addition to its canonical role in delivering $\mathrm{Cu}$ to $\mathrm{Cu}$-dependent secretory enzymes, such as LOX. This role for ATP7A is required for restoring perfusion recovery and neovascularization in ischemic vascular disease.

\section{Results}

Endothelial ATP7A is required for post-ischemic revascularization. To address the role of ATP7A in angiogenesis in vivo, we used the mouse hindlimb ischemia (HLI) model which induces ischemia by femoral artery ligation and excision, as previously reported $^{8,27}$. Immunofluorescence analysis revealed that ATP7A protein expression was robustly increased and colocalized with CD31-positive ECs at day 14 in ischemic muscles compared to nonischemic muscles (Fig. 1a). These results suggest that ATP7A is increased in angiogenic ECs in the HLI model. To determine the role of endogenous ATP7A for ischemia-induced angiogenesis, we used ATP7A ${ }^{\text {mut }}$ mice with reduced ATP7A expression and $\mathrm{Cu}$ transport capacity ${ }^{14}$. Figure $1 \mathrm{~b}, \mathrm{c}$ showed that perfusion recovery and angiogenesis (CD31 positive capillaries), respectively, in response to hindlimb ischemia were significantly impaired in ATP7A $^{\text {mut }}$ mice compared to control, wild type (WT) mice. To determine the relative role of ATP7A in tissue-resident cells and bone marrow (BM) cells in post-ischemic neovascularization, we performed a BM transplantation experiment. Lethally irradiated WT mice transplanted with WT-BM or ATP7A ${ }^{\text {mut }}$ BM showed no significant difference in perfusion recovery after ischemic injury (Fig. 1d), suggesting that ATP7A in tissue-resident cells, but not in $\mathrm{BM}$ cells, is required for ischemia-induced neovascularization.

To determine the role of endothelial ATP7A in ischemiainduced angiogenesis on adult-onset in vivo, we generated inducible EC-specific hemizygous male ATP7A knockout (iECATP7A KO) mice by crossing homozygous floxed females $\left(A T P 7 A^{\mathrm{fl} / \mathrm{fl}}\right)$ with mice expressing tamoxifen-inducible Cre recombinase under the control of the VE-cadherin promoter $\left(\mathrm{ATP}^{+/ / Y} \text {; Cdh5-CreERT2 }{ }^{+/-}\right)^{26}$ (Fig. 1e, f). Selective deletion of ATP7A in ECs was validated by qPCR analysis in ECs and vascular smooth muscle cells (VSMC) isolated from iECATP7A KO mice (Fig. 1g, h). Although global ATP7A KO mice were embryonic lethal ${ }^{12}$, iEC-ATP7A KO mice survived and 

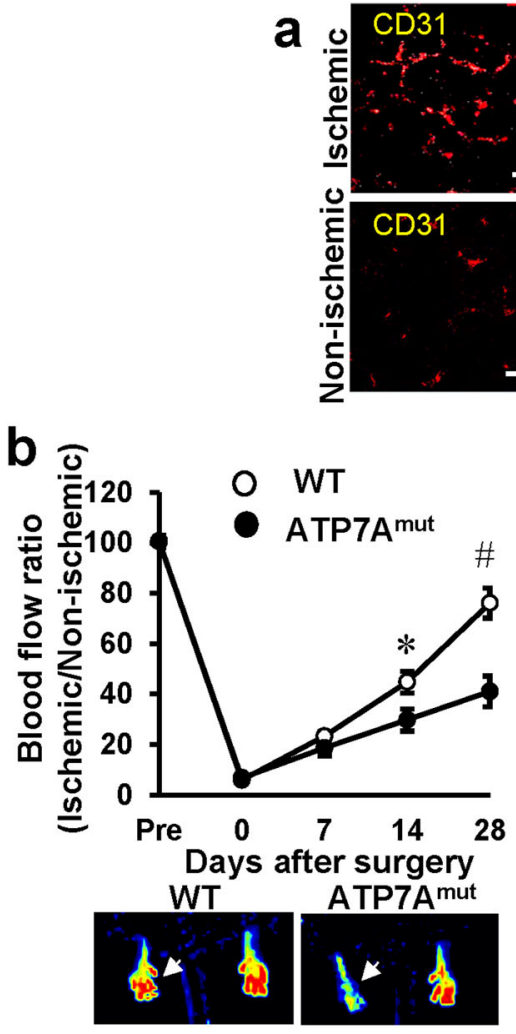

e
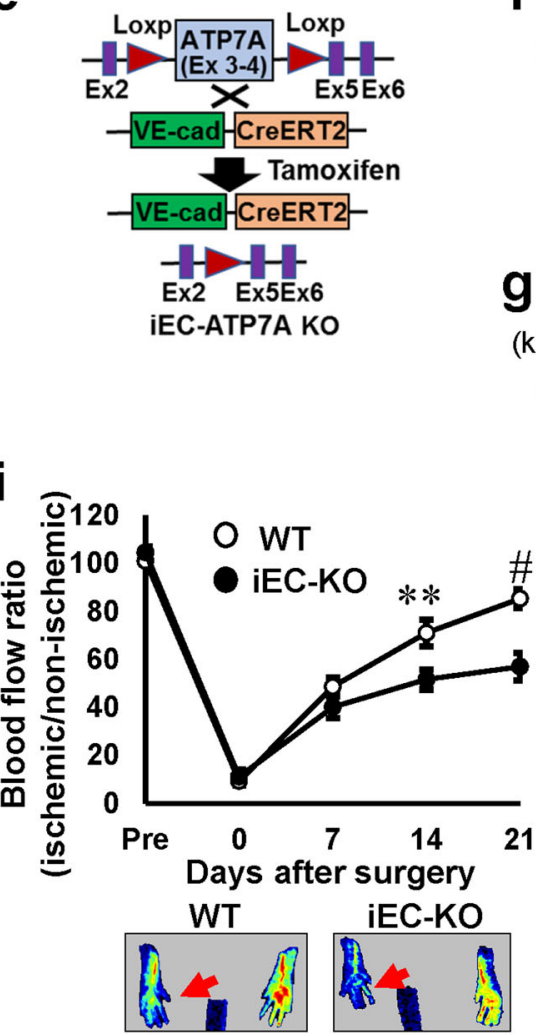

c

$\mathbf{f}$
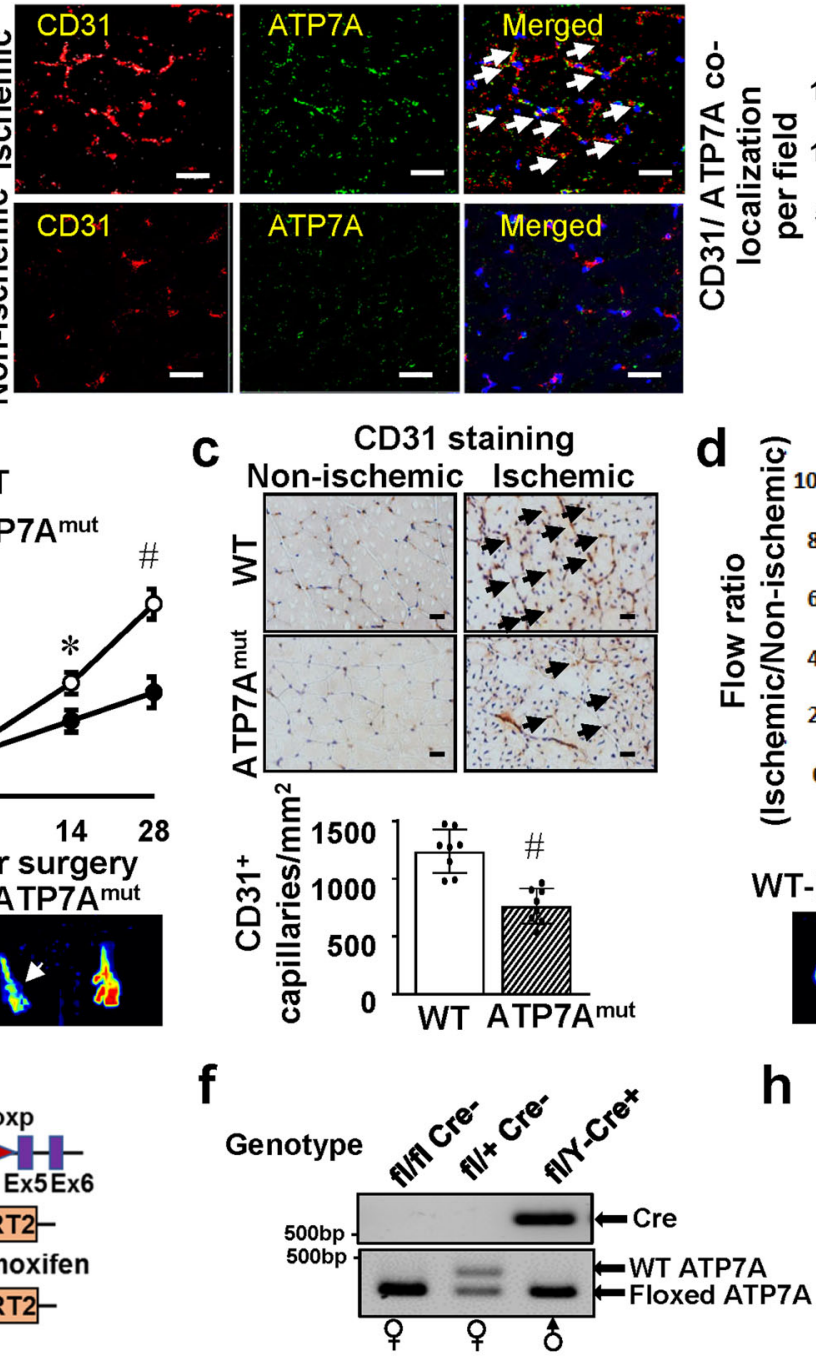

g

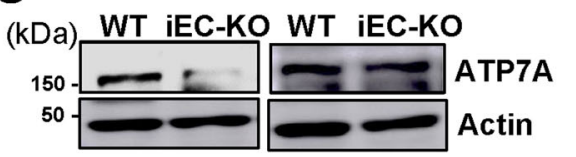

j

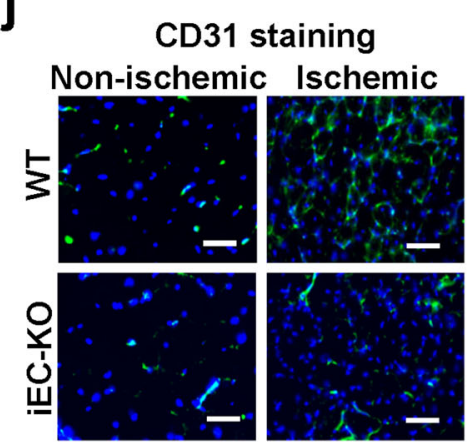

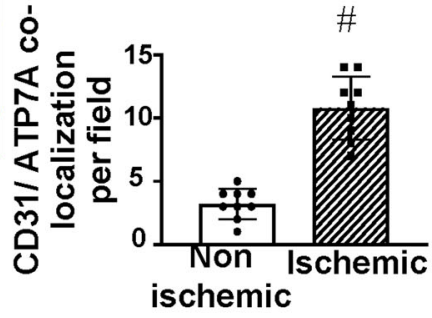
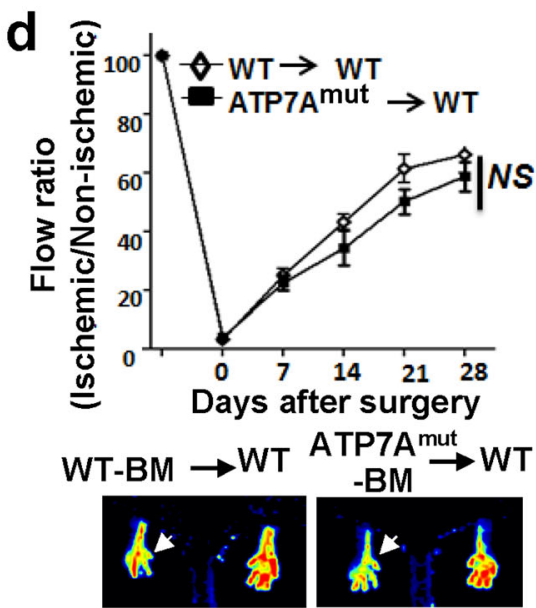

h

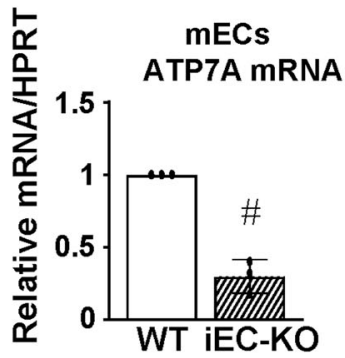

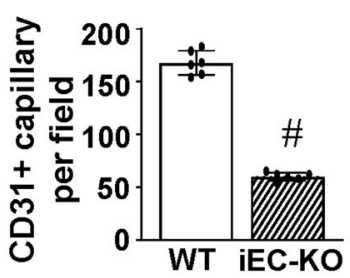

were indistinguishable from WT mice, displaying no obvious abnormalities. Blood flow recovery and $\mathrm{CD} 31^{+}$capillary density in ischemic muscle were significantly impaired in iEC-ATP7A KO mice compared to WT mice (Fig. 1i, j). Thus, endothelial ATP7A plays an important role in post-ischemic neovascularization.
ATP7A knockdown in ECs inhibits VEGFR2 signaling via promotion of VEGFR2 degradation in a Cu-independent manner. We next examined the role of ATP7A in VEGF-induced angiogenic responses in primary cultured ECs. Figure $2 a, b$, using the modified Boyden chamber assay, showed that VEGF-induced EC migration was significantly inhibited in ECs isolated from 


\begin{abstract}
Fig. 1 Endothelial ATP7A is required for ischemia-induced neovascularization. a CD31 (red, Endothelial Cell (EC) marker) or ATP7A (green) staining or their colocalization (merged, white arrows) in ischemic and non-ischemic gastrocnemius muscles at day 14 post-surgery. Scale bars $=50 \mu \mathrm{m}$. The bar graph represents CD31-ATP7A co-localized cell numbers per field. $n=6,{ }_{p} p<0.0001$ (two-tailed unpaired $t$-test). b Blood flow recovery as determined by the ratio of foot perfusion between ischemic (left) and ischemic (right) legs after hindlimb ischemia in Wild Type (WT) and ATP7A mut mice. $n=5$, ${ }^{\star} p<$ $0.05, \# p<0.0001$ (two-way ANOVA followed by Bonferroni's multiple comparison analysis). Bottom panels show representative laser Doppler images (red arrows show ischemic foot) of legs at day 28 after ischemia in WT and ATP7Amut mice. c Capillary density (CD31 staining) in ischemic and nonischemic muscles of WT and ATP7Amut mice at day 14. Scale bars $=50 \mu \mathrm{m}$. Bottom panels show their quantitative analysis (number of capillaries per mm square, $n=8,{ }^{\#} p<0.0001$ (two-tailed unpaired $t$-test). d Bone marrow (BM) from WT or ATP7A mut mice were transplanted to irradiated WT mice. After 6 weeks, mice were subjected to hindlimb ischemia and limb blood flow was measured. $n=6$, NS = non-significant (two-way ANOVA followed by Bonferroni's multiple comparison analysis). The bottom panels show representative laser Doppler images on day 28. e Schematic representation generating inducible EC-specific hemizygous male ATP7A knockout (iEC-ATP7AKO) mice by crossing homozygous floxed females (ATP7Aflfl) with mice expressing the Cre recombinase located downstream of tamoxifen-inducible VE-Cadherin promotor (ATP7A ${ }^{+/ Y}$; Cdh5-CreERT2 ${ }^{+/-}$). f Representative gel

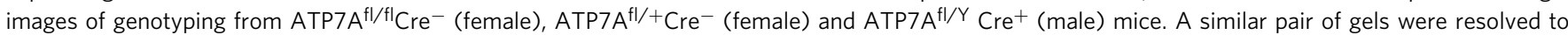
genotype an average of 50 mice/breeding pair with $n=3$ independent breeding pairs. $\mathbf{g}$, $\mathbf{h}$ ATP7A protein ( $\mathbf{g}$ ) and mRNA (h) expression in ECs and aortic vascular smooth muscle cells isolated from WT and iEC-KO mice. $n=3$ animals/group, ${ }^{\#} p=0.0005$ (two-tailed unpaired t-test). $\mathbf{i}$ Blood flow recovery after hindlimb ischemia in WT and iEC-KO mice. The bottom panels show representative laser Doppler images on day $21 . n=5$ animals $/$ group, ${ }^{\star \star} p=0.01$, ${ }^{\#} p<0.0001$ (two-way ANOVA followed by Bonferroni's multiple comparison analysis). $\mathbf{j}$ Capillary density (CD31 staining) in ischemic and non-ischemic muscles of WT and iEC-KO mice at day 14. Right panels show quantification for the number of capillaries per field in ischemic muscles. $n=6,{ }^{\#} p<0.0001$ (two-tailed unpaired $t$-test). Scale bars $=50 \mu \mathrm{m}$. Data are mean $\pm \mathrm{SEM}$.
\end{abstract}

ATP7A ${ }^{\text {mut }}$ mice or HUVECs transfected with ATP7A siRNA. Of note, ATP7A siRNA had no effect on EC migration induced by sphingosine 1-phosphate (S1P), another potent angiogenic Gprotein coupled receptor agonist (Supplementary Fig. 1), supporting the specificity of ATP7A siRNA action. Furthermore, the spheroid EC sprouting assay ${ }^{28}$ showed that ATP7A depletion in ECs significantly inhibited the VEGF-induced increase in the number and length of sprouting from the spheroid (Fig. 2c). These results suggest that ATP7A is specifically involved in angiogenic responses induced by VEGF, but not by S1P in ECs.

To address the mechanism by which ATP7A regulates VEGFinduced angiogenesis, we examined the role of ATP7A in VEGFR2 signaling in ECs. ATP7A knockdown (KD) with siRNA significantly inhibited VEGF-induced VEGFR2 tyrosine phosphorylation and its downstream signalings such as phosphorylation of Akt and p38MAPK, by reducing total VEGFR2 protein (Fig. 2d). Importantly, ATP7A siRNA had no effects on S1Pinduced phosphorylation of Akt or p38MAPK in ECs (Fig. 2e), supporting a specific role of ATP7A in VEGFR2 signaling. Since ATP7A depletion had no effect on VEGFR2 mRNA (Fig. 2f), we next examined whether the decrease in VEGFR2 protein in ATP7A-depleted ECs was due to enhanced degradation or reduced synthesis of VEGFR2. Figure $2 \mathrm{~g}$ showed that VEGFR2 protein expression in the presence of cycloheximide, a eukaryotic protein translation inhibitor, was decreased after VEGF stimulation in a time-dependent manner in both control siRNA and ATP7A siRNA-transfected ECs. These results suggest that ATP7A knockdown in ECs promotes ligand-dependent VEGFR2 protein degradation, which was further confirmed using bovine aortic ECs transfected with two different ATP7A siRNAs (Supplementary Fig. 2). Of note, ATP7A KD in HUVECs had no effect on protein expression of neuropilin-1 (Nrp1), which functions as a VEGFR2 co-receptor ${ }^{29}$ in HUVECs stimulated with VEGF (Supplementary Fig. 3a), nor on ligand-induced degradation of other receptor tyrosine kinases such as Insulin receptor $1 \beta$ (IR1 $\beta$ ) (Supplementary Fig. 3b), fibroblast growth factor receptor 1 (FGFR1) (Supplementary Fig. 3c) or VEGFR3 (Supplementary Fig. 3d) in HUVECs. Thus, ATP7A specifically regulates VEGFR2-mediated signaling and angiogenic responses in ECs.

We then examined if ATP7A KD-induced VEGFR2 degradation was a consequence of $\mathrm{Cu}$ accumulation induced by impaired $\mathrm{Cu}$ exporter (ATP7A) function using tetrathiomolybdate (TTM). TTM is an intracellular $\mathrm{Cu}$ chelator that has been used for the treatment of Wilson Disease and in a number of clinical trials as an anti-tumor agent ${ }^{30-35}$. We found that TTM had no significant effect on either ATP7A KD-induced enhancement of VEGFR2 degradation (Fig. 2h) or inhibition on VEGFR2 signaling (Supplementary Fig. 4a) in HUVECs. The chelator efficacy of TTM was confirmed by showing that TTM almost completely inhibited VEGF-induced Cu-dependent lysyl oxidase (LOX) activity (Supplementary Fig. $4 \mathrm{~b}$ ), as well as $\mathrm{CuCl}_{2}$-induced phosphorylation of ERK, which has been shown to be mediated through direct activation of MEK by $\mathrm{Cu}^{36,37}$ (Supplementary Fig. 4c). These results suggest that loss of ATP7A in ECs inhibits VEGFR2 signaling via promoting VEGFR2 degradation in a $\mathrm{Cu}-$ independent manner.

VEGF stimulation promotes ATP7A binding with VEGFR2. To address the mechanism by which ATP7A stabilizes VEGFR2 protein, we next examined possible interactions between ATP7A and VEGFR2. A co-immunoprecipitation assay showed that VEGF stimulation in ECs rapidly increased ATP7A binding to VEGFR2 within $5 \mathrm{~min}$ in a time-dependent manner (Fig. 3a). In addition, to monitor in situ formation of ATP7A-VEGFR2 complex, we next used the Duolink proximity ligation assay $(\mathrm{PLA})^{38}$, where red puncta indicate positive staining that the epitopes of the target proteins are in close proximity $(<40 \mathrm{~nm})$. We found that both primary mouse ATP7A antibody and rabbit VEGFR2 antibody combined with secondary PLA probes dramatically increased the PLA positive red dots after VEGF stimulation in HUVECs (Fig. 3b). By contrast, single primary antibody only or IgG negative control did not display red dots (Fig. 3b). To confirm further their direct interaction, we performed co-transfection of myc-ATP7A and flag-VEGFR2 plasmids in Cos7 cells and found that myc-ATP7A, but not IgG, was co-immunoprecipitated with flag-VEGFR2 after VEGF stimulation (Fig. 3c). These results suggest the ligand-induced direct interaction between VEGFR2 and ATP7A in situ. In addition, colocalization analysis using confocal microscopy showed that VEGF stimulation promoted ATP7A translocation from TGN to the plasma membrane where it colocalized with VEGFR2 after 5 min, which was followed by co-internalization of ATP7A and VEGFR2 to the perinuclear region after VEGF stimulation for 15 min (Fig. 3d). Taken together, these data show that VEGF stimulation promotes direct binding of ATP7A with VEGFR2 in ECs. 
a

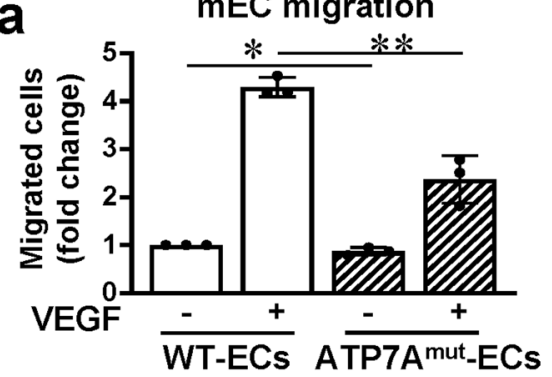

b

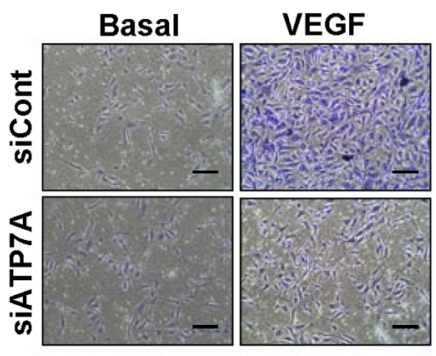

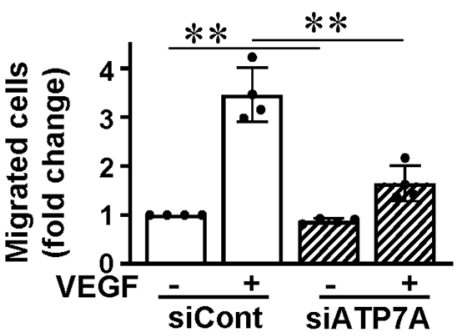

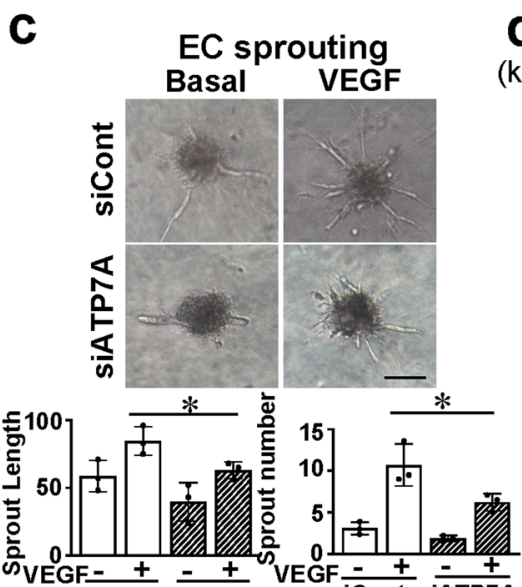

siCont siATP7A

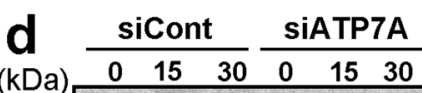

(kDa) $\begin{array}{llllll}0 & 15 & 30 & 0 & 15 & 30\end{array}$

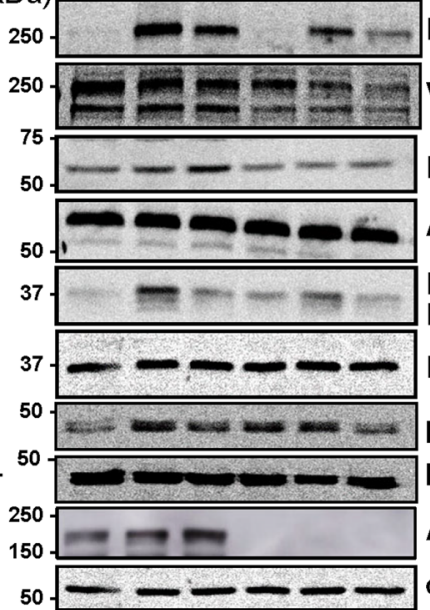

e

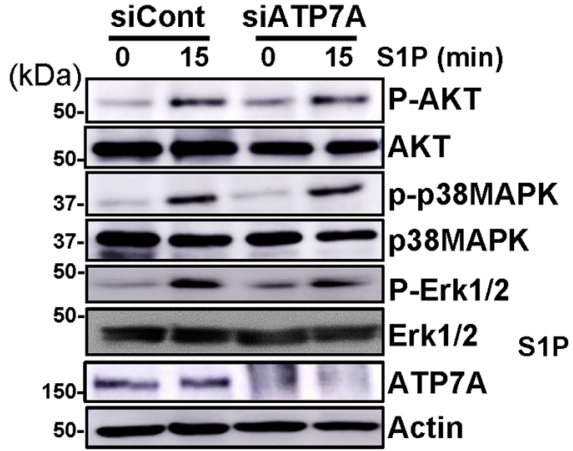

p-VEGFR2

VEGFR2

p-Akt

Akt

p-p38

MAPK

P38MAPK

p-Erk1/2

Erk1/2

ATP7A

$\alpha$-tubulin
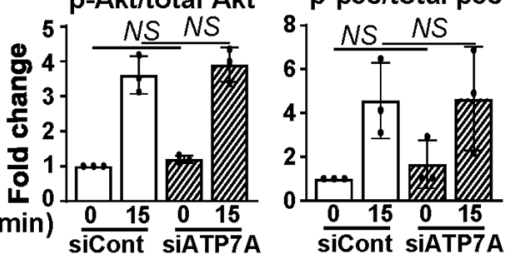
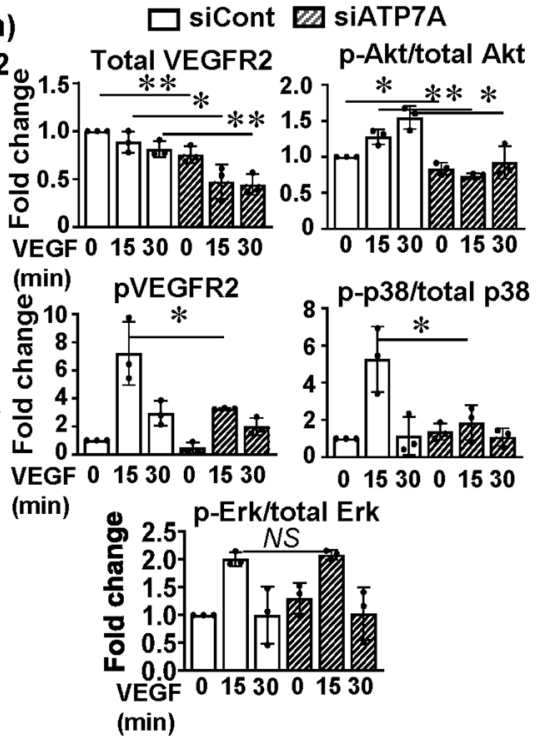

p-Akt/total Akt p-p38/total p38 p-Erk/total Erk

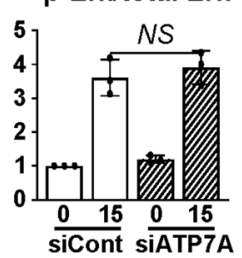

f

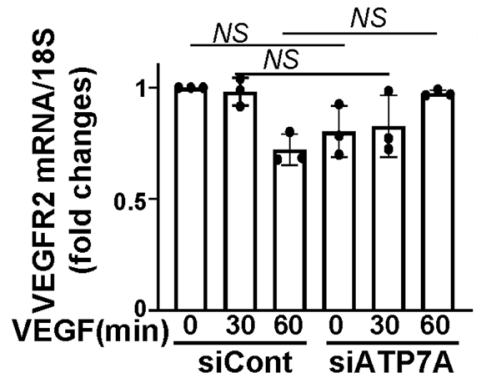

9 Cyclohexamide siCont SiATP7A (kDa) $\begin{array}{lllllll}0 & 15 & 30 \quad 45 & 0 & 15 & 30 \quad 45\end{array}$

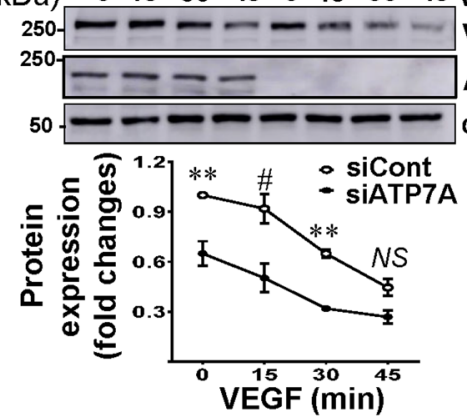

h

sicont siATP7A

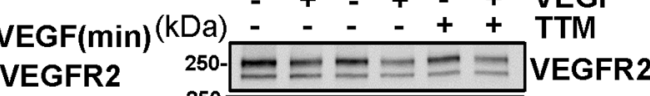
ATP7A $\alpha$-tubulin 250 ATP7A $50-=----$ a-tubulin

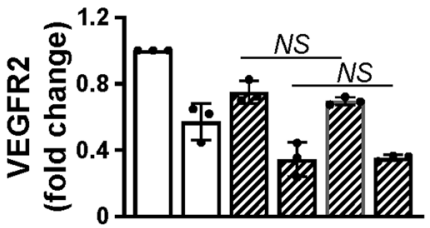

ATP7A functions to limit lysosomal degradation of VEGFR2 in VEGF-stimulated ECs. A cell surface biotinylation assay revealed that VEGF stimulation increased cell surface ATP7A levels within $5 \mathrm{~min}$, followed by a gradual decrease over the next $15 \mathrm{~min}$, which was also associated with a time-dependent reduction of cell surface VEGFR2, reflecting receptor (VEGFR2) internalization (Fig. 4a). Of note, ATP7A knockdown promoted VEGF-induced reduction of cell surface VEGFR2. Interestingly, Fig. 4b, c using co-localization analysis show that VEGF stimulation for 15 min promoted VEGFR2 internalization to Rab5positive early endosomes, but not Lamp2-positive lysosomes, which was followed by trafficking to Rab11-positive recycling 
Fig. 2 ATP7A knockdown inhibits VEGFR2 signaling via promoting VEGFR2 degradation in a Cu-independent manner. $\mathbf{a}$, $\mathbf{b}$ Endothelial cell (EC) migration measured using modified Boyden chamber method in ECs isolated from Wild Type (WT) and ATP7A mut mice (a) or human umbilical vein endothelial cells (HUVECs) transfected with control or ATP7A siRNAs (b) stimulated with vascular endothelial growth factor (VEGF) (20 ng/ml) for $6 \mathrm{~h}$. $\mathbf{a} n=3,{ }^{\star} p=0.0485,{ }^{\star \star} p=0.0034 ; \mathbf{b} n=3,{ }^{\star \star} p=0.0029,{ }^{\star \star} p=0.0016$ (two-tailed unpaired $t$-test). Scale bars $=100 \mu \mathrm{m}$. $\mathbf{c}$ EC spheroid spouting assay in HUVECs transfected with control or ATP7A siRNAs. Right panels show an average number of sprouts and tube length per field. Scale bars $=150 \mu \mathrm{m}$. $n=3$, ${ }^{\star} p=0.038,{ }^{*} p=0.0469$ (two-tailed unpaired $t$-test). d, e HUVECs transfected with control or ATP7A siRNAs were stimulated with VEGF (20 ng/ml) or sphingosine-1-phosphate (S1P) $(10 \mu \mathrm{M})$ for the indicated time. Lysates were immunoblotted (IB) with indicated antibodies (Abs). $\alpha$-tubulin as a loading control. The graph represents the averaged fold change over the basal control. d $n=3$, VEGFR2 ${ }^{\star \star} p=0.0086,{ }^{\star} p=0.0272,{ }^{\star \star} p=0.0092 ; \mathrm{pAKT} /$ total AKT: ${ }^{\star} p=0.0279,{ }^{\star \star} p=0.0011,{ }^{\star} p=0.0177 ; p$-VEGFR2: ${ }^{\star} p=0.0393 ; p$-p38/total $p 38:{ }^{\star} p=0.0413$; NS = non significant; e: $n=3$, NS $=$ non significant (two-tailed unpaired $t$-test). f. HUVECs transfected with control or ATP7A siRNAs were stimulated with VEGF (20 ng/ml) and mRNA level for VEGFR2 normalized by $18 \mathrm{~S}$ was measured. $n=3, \mathrm{NS}=$ non significant (two-tailed unpaired $t$-test). $\mathbf{g}$ HUVECs transfected with control or ATP7A siRNA pretreated with cyclohexamide (10 nM for $10 \mathrm{~min}$ ) were stimulated with VEGF $(20 \mathrm{ng} / \mathrm{ml})$. Lysates were IB with anti-VEGFR2 or $\alpha$-tubulin (loading control) Abs. $n=$ $3,{ }^{\star \star} p<0.01,{ }^{*} p<0.0001$ (two-way ANOVA followed by Bonferroni's multiple comparison analysis). h HUVECs transfected with control or ATP7A siRNAs were pretreated with tetrathiomolybdate (TTM) $(10 \mathrm{nM})$ for $24 \mathrm{~h}$, followed by VEGF stimulation for 30 min. Lysates were IB with anti-VEGFR2 or $\alpha$-tubulin Abs. $n=3, \mathrm{NS}=$ non significant (two-tailed unpaired $t$-test). Data are mean $\pm \mathrm{SEM}$.

endosomes after $30 \mathrm{~min}$. By contrast, in ATP7A-depleted ECs, VEGF stimulation for 15 min facilitated VEGFR2 internalization to Lamp2-positive lysosomes, which was followed by targeting Rab7-positive late endosomes involved in lysosomal degradation after $30 \mathrm{~min}$. These results suggest that ATP7A functions to limit lysosomal degradation of VEGFR2 in VEGF-stimulated ECs. Furthermore, a lysosomal inhibitor (Chloroquine), but not proteasome inhibitors (Lactacystin and MG132), prevented ATP7A KD-induced VEGFR2 degradation (Fig. 4d), suggesting that VEGF-induced ATP7A binding to VEGFR2 may prevent VEGFR2 targeting to lysosomes for degradation. Since autophagy induces the lysosomal degradation of cellular proteins, we next examined the possible role of autophagy in ATP7A KD-induced VEGFR2 degradation. Figure $4 \mathrm{e}$ showed that bafilomycin A1, a vacuolar $\mathrm{H}+$ ATPase (V-ATPase) blocker which inhibits fusion between autophagosomes and lysosomes ${ }^{39}$, prevented the VEGFinduced VEGFR2 degradation in ATP7A-depleted ECs. Furthermore, rapamycin, an autophagy inducer and inhibitor of mTOR $^{40,41,42}$, which increased LC3II expression, promoted reduction of VEGFR2 protein in both basal and VEGF-stimulated HUVECs (Supplementary Fig. 5). Thus, these results suggest that autophagy-dependent mechanisms are involved in the lysosomal degradation of VEGFR2 in ATP7A depleted ECs.

ATP7A depletion induces autophagy and promotes VEGFR2 colocalization with light chain 3 (LC3). To determine whether ATP7A depletion induces autophagy to promote lysosomal degradation of VEGFR2, HUVECs were transfected with a red fluorescent protein (RFP) and green fluorescent protein (GFP) double-tagged LC3 construct which shows the mature and immature LC3-positive puncta ${ }^{43}$ (Fig. 5a). Since the GFP signal, but not the RFP signal, is sensitive to acidic conditions, immature autophagosomes show yellow dots due to intact GFP and RFP signals, while mature autophagolysosomes show only the RFP signal as the green GFP signal is acid-quenched. Figure 5 a showed that $\mathrm{RFP}(+) \mathrm{GFP}(-)$ mature autophagolysosome formation was slightly increased by ATP7A KD in the basal state, which was further enhanced after VEGF stimulation for $30 \mathrm{~min}$ in ECs. In order to examine if ATP7A regulates autophagy during angiogenesis in vivo, we used the hindlimb ischemia model and autophagy reporter CAG-RFP-EGFP-LC3 transgenic mice crossed with or without ATP7A ${ }^{\text {mut }}$ mice. These autophagy reporter mice can detect newly formed autophagosomes with physiological $\mathrm{pH}$ expressing both RFP and EGFP yielding a yellow color, or mature autophagolysosome with acidification expressing RFP with reduced EGFP 44 . As shown in Fig. 5b, there was a significant increase in RFP (+) EGFP (-) LC3 dots in ischemic hindlimbs of ATP7A $^{\text {mut }}$ mice compared to WT mice. This was associated with a significant increase in LC3 protein expression in ischemic tissues of ATP7A ${ }^{\text {mut }}$ mice compared to WT mice (Fig. 5c). Taken together, these results show that the autophagy flux is increased in ATP7A-depleted ECs stimulated with VEGF or in ATP7A dysfunctional mice in response to ischemia in which angiogenesis is impaired. Transmission electron microscopy (TEM) further confirmed an increase in autophagosome and autophagolysosome formation in VEGF-stimulated ATP7A-depleted ECs compared to control ECs (Fig. 5d). Immunofluorescence analysis also demonstrated that ATP7A knockdown promoted the colocalization of autophagy receptor p62/SQSTM1 with LC3 (Fig. 5e), as well as VEGFR2 with LC3 (Fig. 5f) or Lamp2 (Fig. 4b) in HUVECs stimulated with VEGF. Thus, these results suggest that loss of ATP7A induces autophagy, thereby promoting autophagic and lysosomal degradation of VEGFR2.

ATP7A protects VEGFR2 against degradation by preventing p62 binding to VEGFR2. We then examined how VEGFR2 was targeted to the LC3-positive/Lamp2-positive autophagolysosomes in ATP7A-depleted ECs after VEGF stimulation. It has been shown that ligand-induced VEGFR2 ubiquitination facilitates lysosomal degradation of the receptor ${ }^{45}$ and that p62/SQSTM1 brings ubiquitinated cargoes to autophagosome for degradation ${ }^{19}$. We thus examined the role of ATP7A in VEGFR2 ubiquitination and its interaction with p62/SQSTM1. Figure 6a shows that ATP7A depletion in ECs increased VEGFR2 ubiquitination in the basal state, which was further enhanced after VEGF stimulation. This was associated with a significant increase in VEGFR2 colocalization with p62/SQSTM1 in ATP7A-depleted HUVECs after VEGF stimulation (Fig. 6b). Consistent with this, coimmunoprecipitation assays showed that ATP7A knockdown increased VEGFR2 binding to p62/SQSTM1, which was associated with enhanced VEGF-induced VEGFR2 degradation (Fig. 6c). Significantly, ATP7A was not co-immunoprecipitated with p62/SQSTM1 in ECs with or without VEGF stimulation (Supplementary Fig. 6). To confirm further the specific role of ATP7A in preventing VEGFR2 binding with p62/SQSTM1, as well as VEGFR2 degradation, we performed rescue experiments by transfecting human myc-ATP7A plasmid in ATP7A-depleted bovine aortic endothelial cells (BAECs). Figure $6 \mathrm{~d}$ shows that p62 binding to VEGFR2, as well as VEGFR2 downregulation in ATP7A KD ECs stimulated with VEGF, were inhibited by reexpression of myc-hATP7A. These results suggest that ATP7A binding to VEGFR2 may protect against VEGFR2 degradation by preventing p62 binding to VEGFR2.

It is known that the UBA domain of p62 interacts with ubiquitin and ubiquitin-tagged proteins and acts as a selective autophagy receptor to bring the marked proteins for 

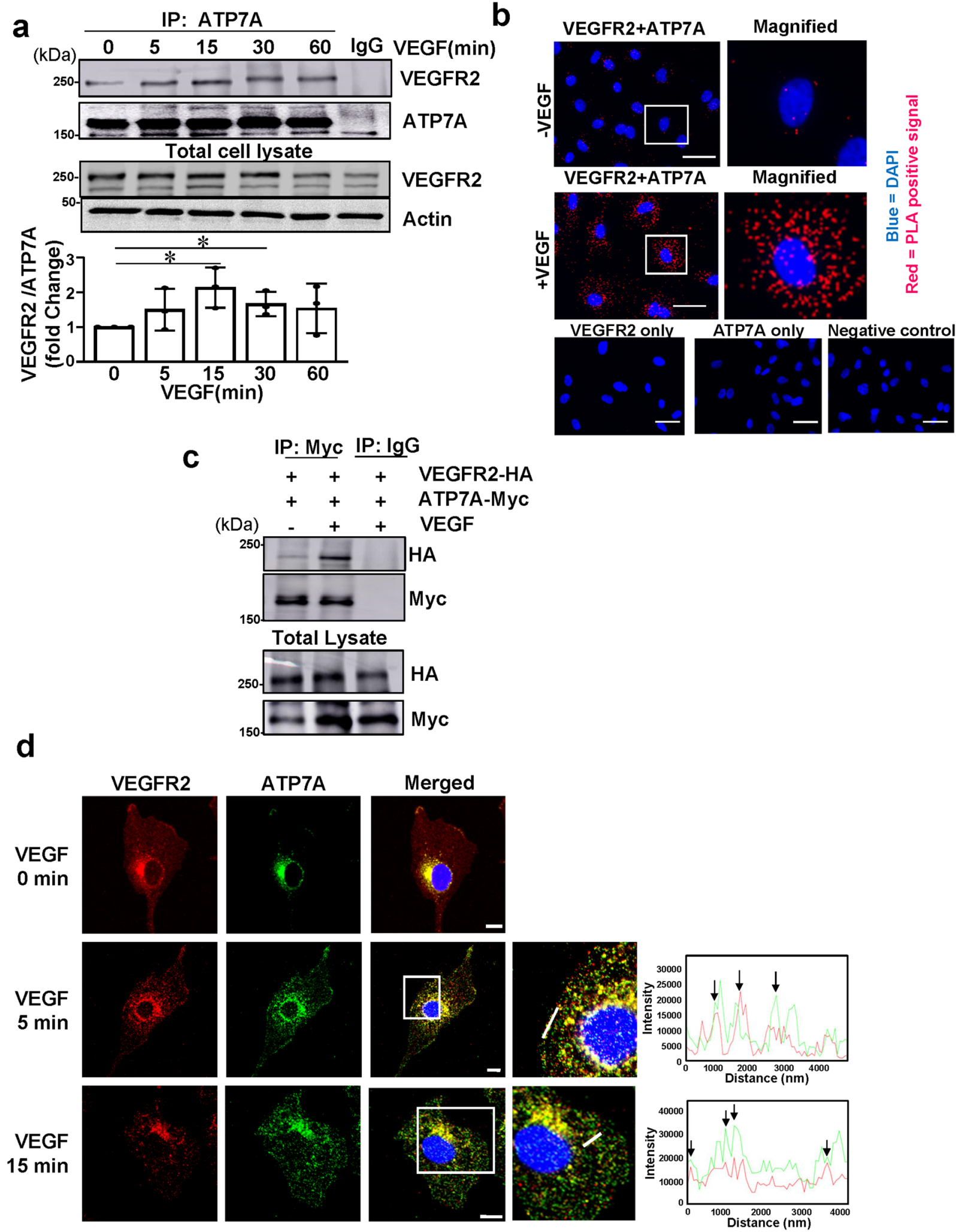

degradation ${ }^{46}$. We next examined the role of the p62 UBA domain in VEGFR2 degradation by transfecting HA-p62-WT or HA-p62 UBA domain deletion mutant (HA-p62 $\Delta$ UBA) in HUVECs. The data in Fig. 6e showed that overexpression of p62WT, but not p62 $\triangle \mathrm{UBA}$, promoted VEGFR2 degradation in ECs with and without VEGF stimulation, as compared to vector control. Furthermore, overexpression of p62 significantly inhibited VEGF-induced EC migration (Fig. 6f). These results suggest that ATP7A protects against autophagic VEGFR2 degradation by preventing VEGFR2 ubiquitination and binding to p62/SQSTM1.

\section{Discussion}

It has been shown that the canonical function of P-type ATPase transporter ATP7A is to deliver $\mathrm{Cu}$ to the secretary $\mathrm{Cu}$ dependent enzymes such as LOX in the TGN and to export 
Fig. 3 VEGF stimulation promotes ATP7A binding with VEGFR2. a Human umbilical vein endothelial cells (HUVECs) were stimulated with vascular endothelial growth factor (VEGF) $(20 \mathrm{ng} / \mathrm{ml})$ for the indicated time. Lysates were immunoprecipitated (IP) with anti-ATP7A antibody (Ab) followed by immunoblotting (IB) with VEGFR2 or ATP7A Ab. The graph represents the averaged fold change of the VEGFR2/ATP7A ratio over the basal ratio. $n=3$, ${ }^{\star} p=0.0275,{ }^{\star} p=0.0303$ (two-tailed unpaired $t$-test). Data are mean \pm SEM. b HUVECs stimulated with VEGF (20 ng/ml) for $30 \mathrm{~min}$ were fixed in $4 \%$ paraformaldehyde. In situ Proximity, Ligation Assay (PLA) was performed to show the interaction of ATP7A with VEGFR2. Red dots indicate PLA positive signal. Either ATP7A or VEGFR2 or no antibody was used as a negative control. The scale bar;10 $\mu \mathrm{m}$. $(n=3)$. c Cos7 cells were transfected with ATP7Amyc and VEGFR2-HA with or without VEGF $(20 \mathrm{ng} / \mathrm{ml})$ stimulation. Lysates were subjected to anti-Myc IP and anti-HA IB. ( $n=3$ ). d Co-localization of VEGFR2 and ATP7A in HUVECs stimulated with VEGF $(20 \mathrm{ng} / \mathrm{ml})$, showing yellow fluorescence in the merged image, was analyzed by comparing the fluorescence intensity for each protein (white line on the enlarged image). Scale bars $=10 \mu \mathrm{m} .(n=3)$.

excess $\mathrm{Cu}$ from the cell ${ }^{5,6}$. This study provides evidence of crosstalk among $\mathrm{Cu}$ transporter ATP7A, the process of autophagy, and VEGFR2 degradation. We show that, in response to VEGF, ATP7A binds to VEGFR2 in ECs and functions to protect against p62/SQSTM1-mediated autophagic and lysosomal degradation of VEGFR2. In this way, ATP7A promotes VEGFR2 signaling, thereby driving angiogenesis (Fig. 7). Thus, endothelial ATP7A is identified as a potential therapeutic target to restore limb perfusion and revascularization in ischemic vascular disease via specific effects on VEGF receptor signaling.

This study demonstrates that ATP7A KD increased VEGFR2 ubiquitination and lysosomal degradation, thereby reducing VEGFR2 signaling. Of note, ATP7A KD had no significant effects on ligand-induced degradation of other tyrosine kinases such as IGFR1, FGFR1, and VEGFR3, or G-protein coupled receptor S1P-induced angiogenic signaling and EC migration in HUVEC. Mechanistically, experiments using co-immunoprecipitation, PLA, and co-transfection assays reveal that VEGF stimulation rapidly induces ATP7A binding to VEGFR2, which promotes VEGFR2 signaling and angiogenesis. It is shown that VEGF binding to VEGFR2 at the cell surface promotes receptor internalization to early endosomes, which is essential for activating sustained VEGFR2 signaling ${ }^{2}$, while some fraction of the receptor is ubiquitinated and sorted to the lysosome for degradation ${ }^{4}$ or else recycled to the plasma membrane ${ }^{47}$. By contrast, ATP7A is shown to be present predominantly at the TGN where it provides $\mathrm{Cu}$ to the secretory $\mathrm{Cu}$ enzymes, but in response to excess $\mathrm{Cu}$, ATP7A translocates from the TGN to the plasma membrane via Rab22a to export $\mathrm{Cu}$ from the cells ${ }^{10,48}$. In this study, immunofluorescence analysis shows that VEGF promotes ATP7A translocation from the perinucleus to the plasma membrane where it colocalizes with VEGFR2 within $5 \mathrm{~min}$, followed by their cointernalization to early endosomes where VEGFR2 signaling is propagated ${ }^{49}$. Consistent with our results, various non-metal stimulants such as insulin, NMDA, PDGF, and hypoxia $17,27,50,51$ have been shown to induce re-localization of ATP7A from the TGN. These results suggest that ATP7A is specifically coupled to VEGFR2-mediated signaling and angiogenesis via binding to VEGFR2 in ECs.

The functional significance of endothelial ATP7A in angiogenesis in vivo is demonstrated utilizing tamoxifen-inducible ECspecific ATP7A deficient mice or ATP7A-dysfunctional ATP7Amut mice which showed impaired post-ischemic neovascularization. We found that ATP7A is highly expressed in $\mathrm{CD}_{3}{ }^{+}$angiogenic ECs in ischemic muscles. Bone marrow transplant experiments showed that ATP7A in tissue-resident cells, but not bone marrow cells, is required for ischemia-induced neovascularization. The process of angiogenesis includes EC migration and capillary tube formation. Consistent with this, ATP7A knockdown in HUVECs significantly inhibited VEGFinduced EC migration (using the modified Boyden chamber assay and wound scratch assay), as well as capillary tube formation using a spheroid EC sprouting assay. In addition, we showed that VEGF-induced EC migration is also inhibited in ECs isolated from ATP7A ${ }^{\text {mut }}$ mice compared to ECs from control mice. Thus, our data provide compelling evidence that ATP7A plays an essential role in VEGF-induced angiogenesis in ECs. The importance of ATP7A in endothelial function is also supported by our previous reports that ATP7A in VSMCs protects against endothelium-dependent vasodilation in hypertensive ${ }^{14,52}$ or diabetic mice ${ }^{16}$. The role(s) of endothelial ATP7A in physiological angiogenesis, such as in embryonic and retinal angiogenesis, as well as in pathological angiogenesis (such as tumor angiogenesis and diabetic retinopathy) needs to be explored in the future studies.

Previous studies show that Ephrin $\mathrm{B} 2^{53}, \mathrm{Nrp}^{54}, \mathrm{NUMB}^{55}$, or scaffold protein, IQGAP1 ${ }^{56}$ bind to VEGFR2 at the cell surface to promote VEGFR2 signaling and angiogenesis. Ephrin B2 is a transmembrane protein that promotes clathrin-dependent VEGFR2 internalization. Mutation of its C-terminal PDZ-binding motif impairs VEGFR2 endocytosis and hence angiogenesis ${ }^{53}$. The presence of a consensus PDZ-binding motif at the $\mathrm{C}$ terminal of ATP7 ${ }^{57}$ suggests its functional similarity with Ephrin B2 in the regulation of VEGFR2 internalization. Nrp1 also binds to VEGFR2 in a ligand-independent manner and promotes VEGFR2 internalization through Rab11 and regulates angiogenesis ${ }^{58}$. However, we found that ATP7A KD promotes ligand-dependent VEGFR2 degradation without altering Nrp1 protein expression. We previously reported that PDGF stimulation in VSMCs promotes ATP7A binding to scaffold protein IQGAP1, which promotes Rac1-dependent VSMC migration and neointimal formation after vascular injury ${ }^{59}$. Thus, ATP7A may bind to the VEGFR2 along with its other binding partners, thereby promoting VEGFR2 signaling and angiogenesis. Addressing this complex binding scenario in detail is beyond the scope of this study, but will be the subject of future work.

It has been reported that VEGFR2 degradation is regulated by ubiquitin ligases $\beta$ TRCP $1^{60}$ or by $\mathrm{Cbl}$ which polyubiquitylates the receptor $^{61}$ and that epsin promotes lysosomal degradation of ubiquitinated VEGFR2 ${ }^{62}$. Thus, ATP7A binding to VEGFR2 may prevent receptor ubiquitination, which in turn may limit epsindependent VEGFR2 endocytosis, leading to lysosomal degradation ${ }^{63}$. Interestingly, to maintain homeostatic balance, newly synthesized VEGFR2 traffics through the Golgi to the plasma membrane to replenish the cell surface pool, in part via Golgi-localized t-SNARE family, syntaxin $6^{64}, \mathrm{KIF} 13 \mathrm{~B}^{65}$, or RNF $121^{66}$. When this pathway is impaired, it facilitates lysosomal degradation of VEGFR2 resulting in inhibition of angiogenesis. Thus, ATP7A depletion at the TGN may prevent newly synthesized VEGFR2 trafficking through the Golgi to the cell surface, thereby facilitating lysosomal degradation. To support this notion, recent studies show that high glucose (as in diabetes) accelerates Golgi-localized VEGFR2 phosphorylation, which promotes VEGFR2 degradation, reducing replenishment of the surface pool ${ }^{67}$.

Autophagy plays an important role in a wide range of physiological processes and human diseases. Kumar et al. ${ }^{68}$ have reported that autophagy induction negatively regulates angiogenesis. In this 


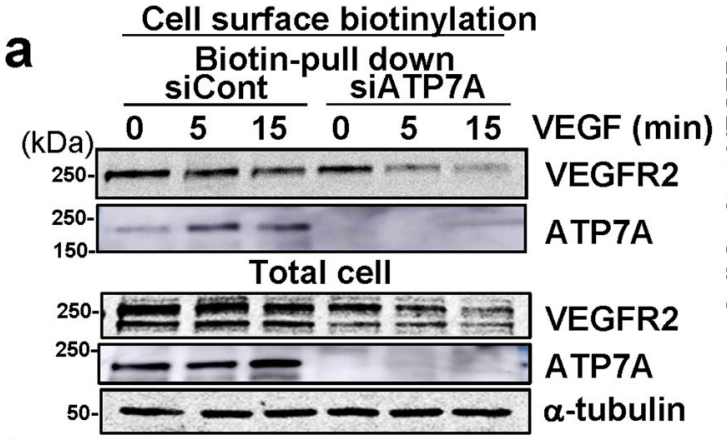

b
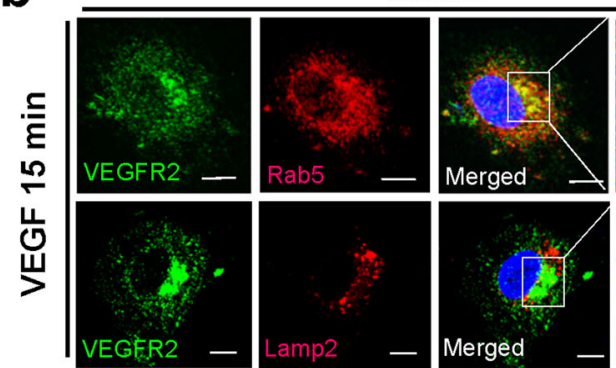

SIATP7A
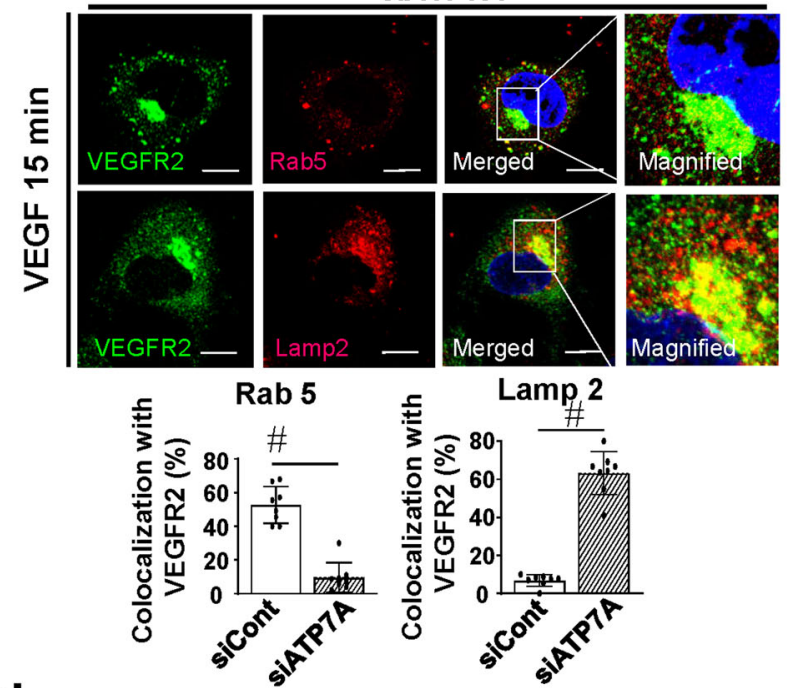

d

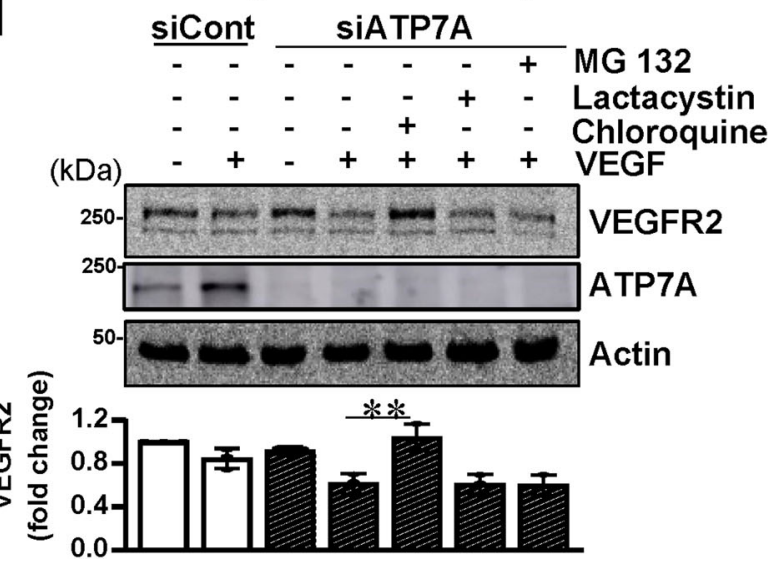

study, experiments using RFP-GFP-LC3 tandem reporter, cell surface biotinylation, immunofluorescence, and TEM, as well as inhibitors of lysosomal or proteasomal protein degradation or autophagy, and the autophagy inducer, rapamycin revealed that loss of ATP7A promotes autophagic flux, including autophagolysosome formation, thereby accelerating VEGFR2 protein degradation.
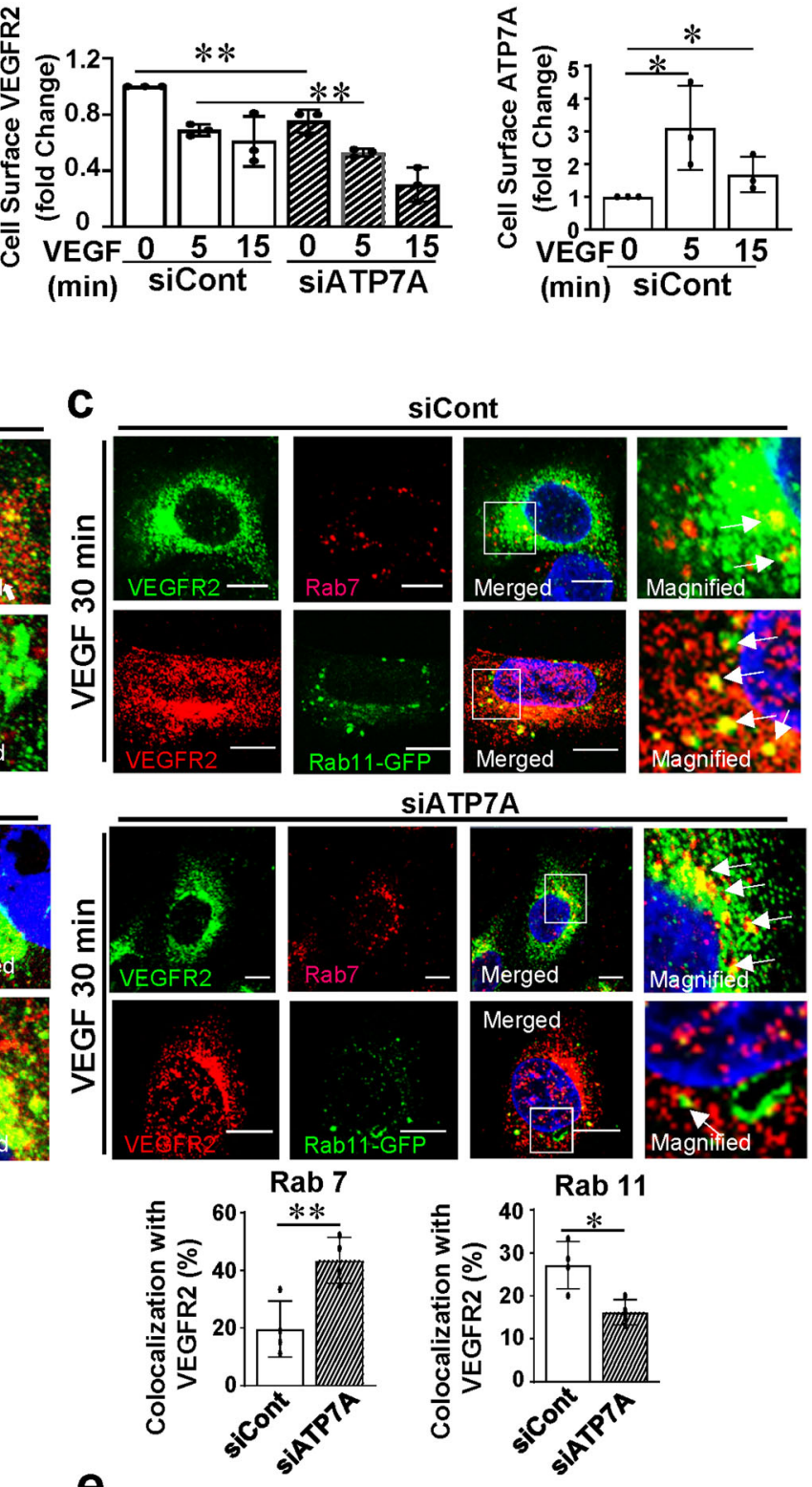

e
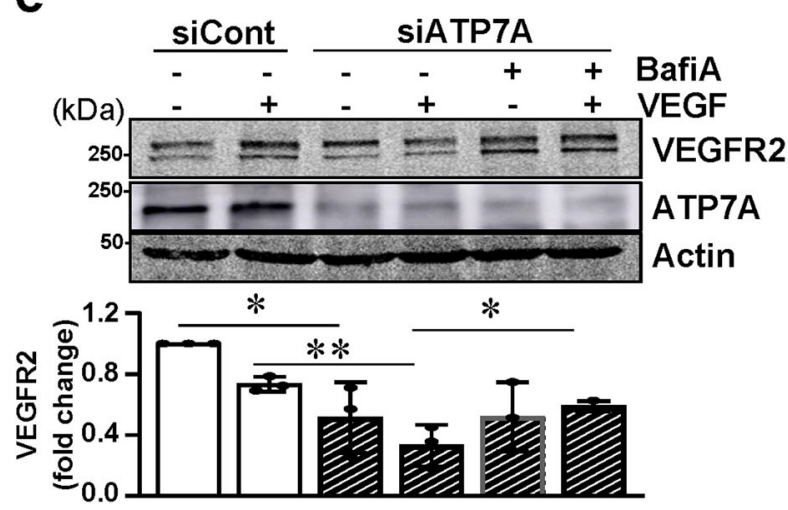

Furthermore, enhanced autophagy flux due to ATP7A dysfunction in vivo was supported by the autophagy reporter CAG-ATP7A ${ }^{\text {mut }}$ RFP-EGFP-LC3 transgenic mice, which was associated with increased expression of autophagy proteins (LC3II), as well as increased RFP(+)EGFP (-)LC3 puncta in ischemic tissues of ATP7A ${ }^{\text {mut }}$ mice. Thus, ATP7A dysfunction apparently induces 
Fig. 4 ATP7A knockdown enhances lysosomal degradation of VEGFR2. a Human umbilical vein endothelial cells (HUVECs) transfected with control or ATP7A siRNAs were stimulated with vascular endothelial growth factor (VEGF) ( $20 \mathrm{ng} / \mathrm{ml}$ ). Lysates were used for cell surface biotinylation using sulfoNHS-SS-biotin to measure cell surface VEGFR2 or ATP7A by biotin pull-down, followed by immunoblotting (IB) with anti-VEGFR2 or ATP7A antibody (Ab). Non-immunoprecipitated (IP) total cell lysate was used for IB with Abs indicated. The bar graph represents the averaged cell-surface VEGFR2 or ATP7A protein levels over the basal control. $n=3$, VEGFR2: ${ }^{\star \star} p=0.0066,{ }^{\star \star} p=0.0046$; ATP7A: ${ }^{\star} p=0.047,{ }^{\star} p=0.0343$ (two-tailed unpaired $t$-test). b HUVECs transfected with control or ATP7A siRNAs stimulated with VEGF ( $20 \mathrm{ng} / \mathrm{ml})$ for $15 \mathrm{~min}$ were used for co-localization analysis for VEGFR2 and Rab5 or Lamp2. Yellow fluorescence in merged images indicates their colocalization. Scale bars $=10 \mu \mathrm{m}$. The bar graph represents the percentage of colocalization with VEGFR2. $n=8$, Rab5: ${ }^{p} p<0.0001$; lamp2: ${ }^{*} p<0.0001$ (two-tailed unpaired $t$-test). c HUVECs transfected with control or ATP7A siRNAs and stimulated with VEGF $(20 \mathrm{ng} / \mathrm{ml})$ for 30 min were used for co-localization analysis for VEGFR2 and Rab7 using the corresponding antibodies. For VEGFR2-Rab11 colocalization, Rab11 was detected through Rab11-GFP plasmid over-expression and VEGFR2 was detected through an anti-VEGFR2 antibody. Yellow fluorescence in merged images indicates their colocalization. Scale bars $=10 \mu \mathrm{m}$. The bar graph represents the percentage of colocalization with VEGFR2. $n=4$, Rab7: ${ }^{\star *} p=0.009$; Rab11: ${ }^{\star} p=0.0128$ (two-tailed unpaired $t$-test). d, e HUVECs transfected with control or ATP7A siRNAs were pretreated with lysosome inhibitor chloroquine ( $500 \mu \mathrm{M}$ for $30 \mathrm{~min}$ ) or proteasome inhibitors MG132 (carbobenzoxy-Leu-Leu-leucinal) (10 $\mu \mathrm{M}$ for $30 \mathrm{~min}$ ) for $30 \mathrm{~min}$, or lactacystin $(10 \mu \mathrm{M}$ for $60 \mathrm{~min}$ ) (d) or bafilomycin A1 ( $5 \mathrm{nM}$ for $60 \mathrm{~min}$ ) (e) followed by VEGF stimulation for 30 min. Lysates were IB with anti-VEGFR2 or actin loading control. $\mathbf{d} n=3,{ }^{\star \star} x=0.009 ; \mathbf{e} n=3,{ }^{\star} p=0.0223,{ }^{\star \star} p=0.0027,{ }^{\star} p=0.0122$ (two-tailed unpaired $t$-test). Data are mean \pm SEM

autophagy, thereby inhibiting post-ischemic neovascularization. Interestingly, it has been reported that the autophagic-lysosomal pathway is essential for ATP7A-mediated and ATP7B-mediated removal of excess $\mathrm{Cu}$ to protect against $\mathrm{Cu}$-mediated toxicity in hepatocytes 23,24 or in senescent mice fibroblasts ${ }^{25}$, indicating a linkage among autophagy, lysosomes, and $\mathrm{Cu}$ transport proteins for regulating $\mathrm{Cu}$ homeostasis. A recent report shows that $\mathrm{Cu}$ binds to autophagic kinases ULK1/2 to regulate their activity, leading to autophagosome complex formation and autophagic flux, which drives cell growth and survival in lung adenocarcinoma ${ }^{69}$.

Our study suggests that ATP7A KD-induced autophagic VEGFR2 degradation is $\mathrm{Cu}$-independent since the $\mathrm{Cu}$ chelator TTM is without effect on this response. It should be noted that the role of autophagy seems to differ, depending on the context of angiogenesis in physiological and pathological models, which is consistent with the notion that autophagy acts as a double-edged sword to regulate angiogenesis. For example, Sprott et al. ${ }^{70}$ reported that EC-specific ATG5-deficient mice show reduced hypoxia/reoxygenation-triggered neovascularization in the retinopathy of prematurity model, suggesting that autophagy is required for pathological angiogenesis. On the other hand, it has been shown that autophagy impaired angiogenesis in vitro and in vivo $^{22}$. We previously demonstrated that ATP7A protein expression is significantly reduced in type 1 and type 2 diabetic vessels $^{16,17}$. Thus, it is possible that ATP7A downregulation in diabetes may contribute to decreased VEGFR2 expression via inducing autophagy, leading to impaired angiogenesis in diabetic peripheral arterial disease. ATP7A downregulation may be an important issue in this pathology and will be the subject of future investigation.

The autophagic cargo/adapter p62/SQSTM1 is known as an adapter protein that delivers cargo and the substrates for autophagic degradation ${ }^{71}$. Recent studies have found that $\mathrm{p} 62$ interacts with ubiquitin and ubiquitin-tagged proteins via the UBA domain and acts as a selective autophagy receptor to delivers polyubiquitinated proteins to the autophagosome by interacting with the autophagosomal membrane protein, LC3. The sequestered cargo is subsequently degraded by lysosomal enzymes when the autophagosome fuses with a lysosome ${ }^{72}$. In this study, we show that loss of ATP7A increased VEGFR2 ubiquitination and recruitment of p62 to the VEGFR2 and their colocalization with LC3, leading to accelerated autophagic degradation of VEGFR2. The functional significance of $\mathrm{p} 62$ / SQSTM1 is demonstrated by the result that overexpression of p62 reduced the VEGFR2 protein expression and VEGF-induced EC migration while p62 UBA domain deletion mutant had no effects on VEGFR2 expression (see Fig. 6e). Consistent with our results, a previous study showed that p62 is involved in the starvation-induced autophagic degradation of IRS-1 ${ }^{73}$. Of note, we found that VEGF stimulation promotes ATP7A binding to VEGFR2, but not to p62 in ECs (Fig. 3 and Supplementary Fig. 6). These observations suggest that ATP7A prevents interaction between the ubiquitinated VEGFR2 and the p62/SQSTM1 via binding to VEGFR2, thereby limiting the autophagic degradation of VEGFR2, which in turn promotes VEGF-induced angiogenesis in ECs. Given that p62 is a multifunctional scaffolding protein that interacts with various proteins to regulate diverse processes including apoptosis, as well as redox state via regulating the KEAP1-NRF2 pathway ${ }^{74}$, other p62-mediated mechanisms may be involved in p62-induced impaired angiogenic responses. Further studies are necessary to understand the other mechanisms by which ATP7A dysfunction induces autophagy in ECs or other cell types.

The canonical role of ATP7A is to deliver $\mathrm{Cu}$ to the secretory $\mathrm{Cu}$ dependent enzymes including SOD3 and LOX to regulate its activity ${ }^{5,6}$. We and others showed that LOX promotes tumorigenesis and metastasis ${ }^{7}$ and is partially involved in VEGF-induced angiogenesis in $\mathrm{ECs}^{8,9}$. We reported that SOD3 is required for postischemic neovascularization ${ }^{9}$. Surprisingly, we found that the $\mathrm{Cu}$ chelator TTM ${ }^{30-34,51,75}$ had no effect on either ATP7A depletioninduced VEGFR2 degradation of VEGFR2 signaling. Of note, the chelator efficacy of TTM was confirmed by showing that TTM almost completely inhibited VEGF-induced $\mathrm{Cu}$-dependent LOX activity, as well as $\mathrm{CuCl}_{2}$-induced phosphorylation of ERK, which is downstream of MEK which is directly activated by $\mathrm{Cu}^{36,37}$. These results suggest that the $\mathrm{Cu}-\mathrm{LOX}$ axis-dependent ATP7A-mediated angiogenesis may be downstream of ATP7A-mediated $\mathrm{Cu}$-independent regulation of VEGFR2 signaling. In previous studies, we demonstrated that ATP7A in VSMCs plays an important role in PDGF-induced vascular migration in part via delivering $\mathrm{Cu}$ to $\mathrm{LOX}^{27,59}$; however, a role for ATP7A in regulating PDGFR expression levels in VSMC was not investigated. In ECs, since ATP7A KD had no significant effects on ligand-induced degradation of other tyrosine kinases and S1P-induced angiogenic signaling and EC migration, ATP7A seems not to be involved in all angiogenic responses.

In summary, our study demonstrates the endothelial ATP7Aautophagy linkage in regulating VEGFR2 signaling and provides evidence that ATP7A functions to promote VEGF-induced angiogenesis via limiting autophagic VEGFR2 degradation, in addition to its canonical function in delivering $\mathrm{Cu}$ to secretory $\mathrm{Cu}$ enzymes. These findings provide insights into endothelial ATP7A as a potential therapeutic target for the treatment of ischemic cardiovascular diseases. 
a
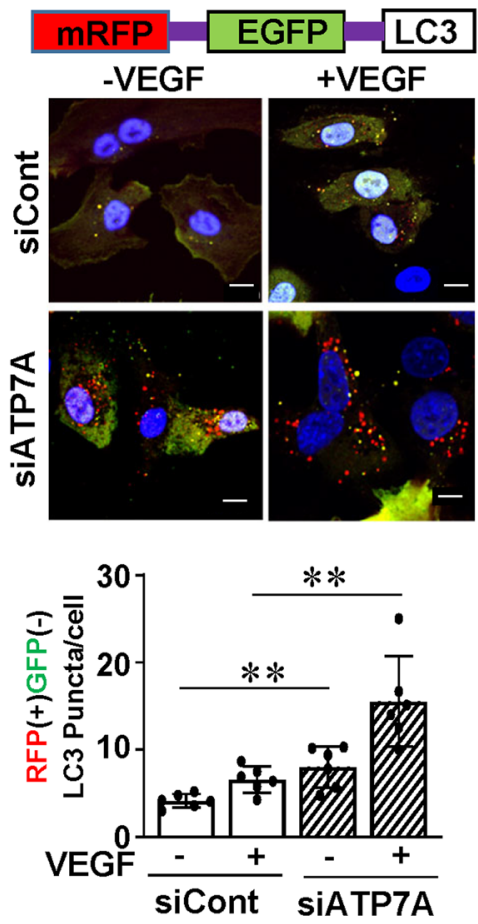

d

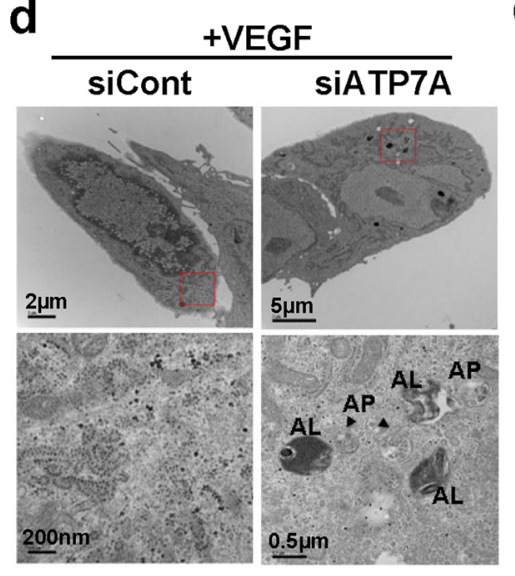

b
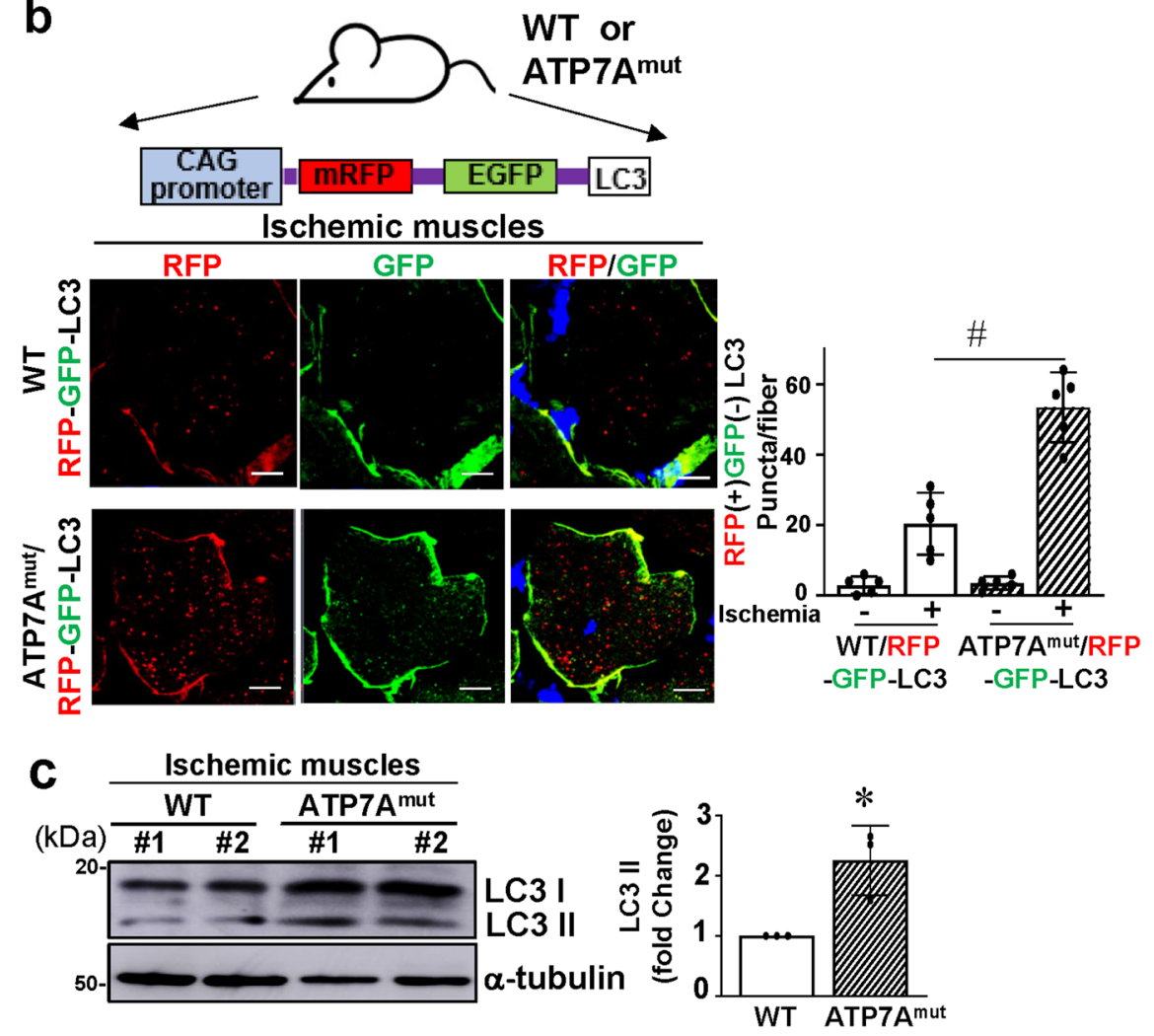

e

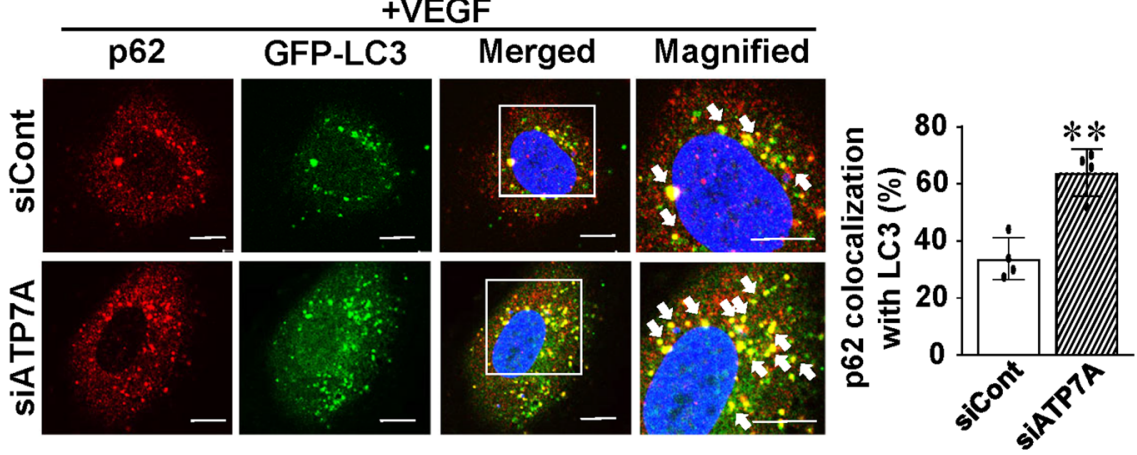

$f$

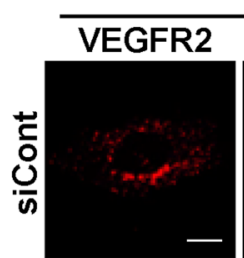

$+\mathrm{VEGF}$
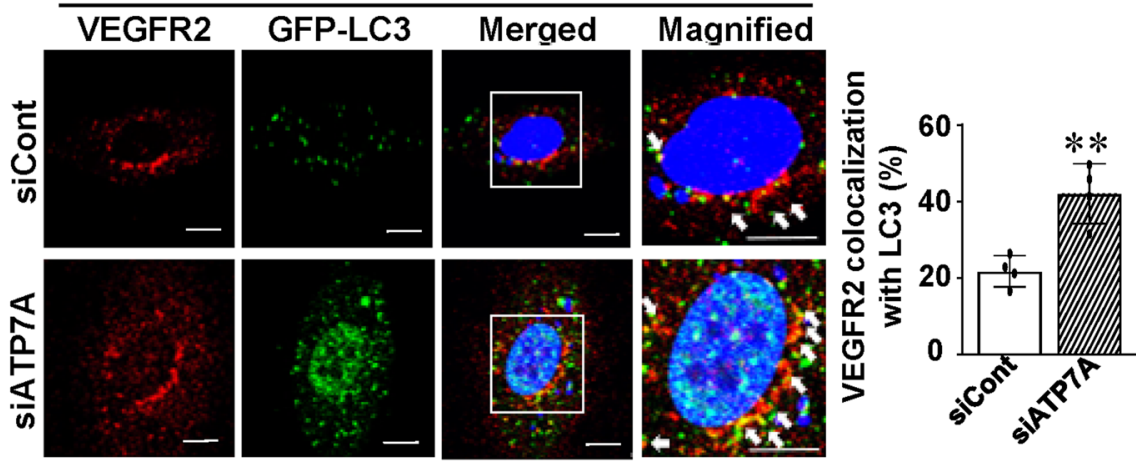

\section{Methods}

Animals. The use of mice was in accordance with the National Institutes of Health Guide for the Care and Use of Laboratory Animals and relevant ethical regulations. Mouse experiments were approved by the Institutional Animal Care and Use Committee of Augusta University. To generate EC-specific ATP7A KO mice, we first generated an ATP7A floxed mouse line using ATP7A floxed ES cells obtained from European Conditional Mouse Mutagenesis Program (EUCOMM). We removed the NEO resistance gene by breeding with Flp recombinase expressing transgenic mouse. Inducible EC-specific hemizygous male ATP7A knockout (iECATP7A KO) mice were generated by crossing homozygous floxed females

$\left(\mathrm{ATP} 7 \mathrm{~A}^{\mathrm{fl} / \mathrm{fl}}\right)$ with mice expressing tamoxifen-inducible Cre recombinase under the control of the VE-cadherin promoter (ATP7A ${ }^{+/ Y} ;$ Cdh5-CreERT2 ${ }^{+/-}$). ATP7A deletion was initiated by daily intraperitoneal injections of $40 \mu \mathrm{g}$ tamoxifen (SigmaAldrich)/g body weight for 10 days with 2 days break. Control mice, negative for 
Fig. 5 ATP7A depletion induces autophagy and promotes VEGFR2 colocalization with LC3. a Immunofluorescence analysis of LC3 (Microtubuleassociated protein 1A/1B-light chain 3)-RFP and GFP puncta (mature and immature LC3 puncta, respectively), human umbilical vein endothelial cells (HUVECs) transfected with LC3-RFP-GFP plasmids in the presence of either ATP7A or control siRNAs were treated with vascular endothelial growth factor (VEGF) $(20 \mathrm{ng} / \mathrm{ml})$ for $30 \mathrm{~min}$. Scale, $10 \mu \mathrm{m}$. The bar graph represents averaged number of RFP positive and GFP negative puncta per cell. $n=6,{ }^{\star \star} p=$ $0.0033,{ }^{\star \star} p=0.0022$ (two-tailed unpaired $t$-test). b. ATP7A ${ }^{\text {mut }}$ mice crossed with RFP-EGFP-LC3 transgenic mice to generate ATP7A ${ }^{\text {mut }} /$ RFP-EGFP-LC3 transgenic reporter mice. Wild Type (WT) and ATP7Amut reporter mice were subjected to hind limb ischemia. Gastrocnemius muscles were harvested at 3 days after ischemia and examined for RFP and GFP expression. The scale bar; $10 \mu \mathrm{m}$. Bar graph represents averaged number of RFP positive and GFP negative puncta per fiber. $n=5,{ }^{\#} p=0.0005$ (two-tailed unpaired $t$-test). c Representative western analysis of ischemic gastrocnemius muscles of WT and ATP7A ${ }^{\text {mut }}$ mice at 3 days after ischemia. The bar graph represents averaged fold change over control and tubulin as a loading control. $n=3$, ${ }^{\star} p=$ 0.0196 (two-tailed unpaired $t$-test). $\mathbf{d}$ HUVECs transfected with control or ATP7A siRNAs were stimulated with VEGF (20 ng/ml) for $30 \mathrm{~min}$ and were analyzed by transmission electron microscopy. Double membrane indicates autophagosome (AP) and black vesicle included AP indicated autolysosome (AL). ( $n=3)$. e, $\mathbf{f}$ HUVECs transfected with LC3-GFP plasmid in the presence of either ATP7A or control siRNAs were stimulated with VEGF (20 ng/ml) for $30 \mathrm{~min}$. Cells were stained with anti p62 or VEGFR2 antibodies. The right panels depict magnified images of the boxed areas seen in the left panels. The scale bar: $10 \mu \mathrm{m}$. Bar graph represents averaged number of p62 or VEGFR2 and LC3 colocalized puncta per cell. e $n=4,{ }^{\star \star} p=0.0016 ; \mathbf{f} n=4{ }^{\star \star} p=0.0036$ (two-tailed unpaired $t$-test). Data are mean \pm SEM.

Cre expression received the same treatment. The mice were allowed to recover for 7 days after the last tamoxifen treatment before experiments. CAG-RFP-EGFP-LC3 transgenic mice expressing RFP and EGFP in a pH-dependent manner were bought from Jackson Laboratory (Stock No:027139). In some experiments, we used hemizygous male blotchy ATP7A mutant (ATP7A $\left.{ }^{\text {mut }}\right)$ mice, which were generated by backcrossing ATP7A ${ }^{\text {mut }}$ mice on C57BL/6JEiJ background (Jackson Laboratory

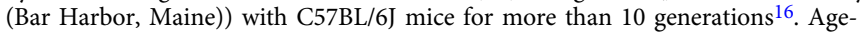
matched C57BL6 mice used for control, wild-type (WT) mice, were purchased from Jackson Laboratory. ATP7A ${ }^{\text {mut }}$ mice carrying the X-linked blotchy ATP7A mutation have a splice site mutation introducing a new stop codon at amino acid residue 794 and show impaired copper transport function but survive to more than 6 months of age. All mice were maintained at the Augusta University animal facility. Room temperature and humidity were maintained at $22.5^{\circ} \mathrm{C}$ and between $50 \%$ and $60 \%$, respectively. All mice were held under the 12:12 (12-h light:12-h dark) light/dark cycle. Mice were held in individually ventilated caging with a maximum of 5 or a minimum of 2 mice per cage. Mice at 8 to 12 weeks old were used for experiments.

Hindlimb ischemia model. Mice were subjected to unilateral hindlimb surgery under anesthesia with intraperitoneal administration of ketamine $(87 \mathrm{mg} / \mathrm{kg})$ and xylazine $(13 \mathrm{mg} / \mathrm{kg})$. We performed ligation and segmental resection of left femoral vessels followed by physiological and histological analysis ${ }^{76}$. Briefly, the left femoral artery was exposed, ligated both proximally and distally using 6-0 silk sutures and the vessels between the ligatures were excised without damaging the femoral nerve. All arterial branches between the ligations were obliterated using an electricalcoagulator (Fine Scientific Tools). Skin closure was done using 6-0 nylon sutures. We measured ischemic (left)/nonischemic (right) limb blood flow ratio using a laser Doppler blood flow (LDBF) analyzer (PeriScan PIM 3 System; Perimed). Mice were anesthetized and placed on a heating plate at $37^{\circ} \mathrm{C}$ for $10 \mathrm{~min}$ to minimize temperature variation. Before and after surgery, LDBF analysis was performed in the plantar sole. Blood flow was displayed as changes in the laser frequency, represented by different color pixels, and mean LDBF values were expressed as the ratio of ischemic to nonischemic LDBF.

Bone marrow (BM) transplantation. BMCs were isolated by density gradient separation. Recipient mice were lethally irradiated with $9.5 \mathrm{~Gy}$ and received an intravenous injection of 3 million donor bone marrow cells $24 \mathrm{~h}$ after irradiation. To determine the transplantation efficiency, transplantation was performed between WT mice (Jackson Laboratories) and either ATP7A ${ }^{\text {mut }}$ or WT mice. Hindlimb ischemia was induced 6 to 8 weeks after bone marrow transplantation.

Cell culture. The primary HUVECs (Human Umbilical Vein Endothelial Cells) purchased from Lonza (CC-2519, USA) were cultured in EndoGRO (EMD Millipore) with 5\% fetal bovine serum (FBS) (Atlanta Biological) until passages 6 . Bovine aortic endothelial cells (BAEC; VEC Technologies) and COS-7 cells (ATCC ${ }^{\circledR}$ CRL-1651 ${ }^{\mathrm{m}}$ ) were grown in Dulbecco's modified Eagle's medium (DMEM) containing 10\% (vol/vol) fetal bovine serum and used for experiments until passage 10

Histological analysis. For cryosections, mice were euthanized and perfused through the left ventricle with saline and $4 \%$ paraformaldehyde, limbs were fixed in $4 \%$ paraformaldehyde (PFA) overnight and incubated with $30 \%$ sucrose, and adductor and gastrocnemius muscles were embedded in OCT compound (Sakura Finetek). For paraffin sections, we performed methanol fixation or PFA fixation with decalcification by Immunocal (Decal Chemical Corp.). Capillary density in the ischemic muscles was determined in $7 \mu \mathrm{m}$ cryosections or in $5 \mu \mathrm{m}$ methanol fixed paraffin sections that were stained with anti-mouse CD31 antibody (BD)(1:300). For immunohistochemistry, we used R.T.U. Vectorstain Elite (Vector Laboratories) followed by DAB visualization (Vector Laboratories). Images were captured by Axio scope microscope (Zeiss) or confocal microscopy (Zeiss) and processed by AxioVision 4.8 or LSM510 or ZEN 2.3 software (Zeiss).

Immunofluorescence analysis. Immunofluorescence staining was performed in fresh frozen OCT tissue sections with primary antibodies against ATP7A (LS Bioscience)(1:300) and CD31 (BD Bioscience) (1:300). Secondary antibodies were Alexa Fluor 488 or 546 -conjugated goat anti-mouse IgG and goat anti-rat IgG (Invitrogen)(1:500). Tissue sections were mounted using Vectashield (Vector Laboratories) containing DAPI for nuclear counter-staining. For cultured cells, HUVECs on glass coverslips were rinsed quickly in ice-cold PBS, fixed in freshly prepared $4 \%$ paraformaldehyde in PBS for $10 \mathrm{~min}$ at room temperature, permeabilized in $0.05 \%$ Triton X-100 in PBS for $5 \mathrm{~min}$, and rinsed sequentially in PBS, $50 \mu \mathrm{mol} / \mathrm{L} \mathrm{NH} \mathrm{N}_{4} \mathrm{Cl}$ and PBS for $10 \mathrm{~min}$ each. After incubation for $1 \mathrm{~h}$ in blocking buffer (PBS $+3 \%$ BSA), cells were incubated with primary antibody for $18 \mathrm{~h}$ at $4{ }^{\circ} \mathrm{C}$, rinsed in PBS/BSA, and then incubated in Alexa Fluor 488-conjugated IgG for $1 \mathrm{~h}$ at room temperature, and cells rinsed with PBS. Cells on coverslips were mounted onto glass slides using Vectashield (Vector Laboratories) and observed using confocal microscopy.

Immunoprecipitation and Immunoblotting. Cells were lysed in lysis buffer, $\mathrm{pH}$ 7.4 (in $\mathrm{mM}$ ) 50 HEPES, 5 EDTA, $120 \mathrm{NaCl}$ ), $1 \%$ Triton X-100, protease inhibitors ( $10 \mu \mathrm{g} / \mathrm{ml}$ aprotinin, $1 \mathrm{mmol} / \mathrm{L}$ phenylmethylsulfonyl fluoride, $10 \mu \mathrm{g} / \mathrm{ml}$ leupeptin) and phosphatase inhibitors $(\mathrm{mmol} / \mathrm{L}) 50$ sodium fluoride, 1 sodium orthovanadate, 10 sodium pyrophosphate). For immunoprecipitation, cell lysates $(1,000 \mu \mathrm{g})$ were precipitated with antibody overnight at $4^{\circ} \mathrm{C}$ and then incubated with $20 \mu \mathrm{l}$ of protein A/G-agarose beads for $1.5 \mathrm{~h}$ at $4{ }^{\circ} \mathrm{C}$. Cell lysates $(25 \mu \mathrm{g})$ or immunoprecipitates were separated using SDS-polyacrylamide gel electrophoresis. Goat antiVEGFR2 (R\&D System), Mouse anti-p62 (BD), and Mouse anti- $\alpha$-tubulin (Santa Cruz) were incubated on nitrocellulose membranes containing protein with 1:1000 dilution. For protein expression in ischemic muscle, mice were perfused with cold phosphate buffer saline. Muscle samples were harvested and frozen in liquid nitrogen. Muscle samples were crushed and lysed with RIPA lysis buffer (5 mM Tris-HCl (pH 7.6), $150 \mathrm{mM} \mathrm{NaCl}, 1 \% \mathrm{NP}-40,1 \%$ sodium deoxycholate, $0.1 \%$ SDS) with protease inhibitor followed by brief sonication. An equal amount of protein was separated by SDS-PAGE. Following primary antibodies were used: antiATP7A (LS Bioscience) and anti-LC3 (MBL) at 1:1000 dilution. The other antibodies used in this study are anti-VEGFR3 (1:1000) from the R\&D System, antiAKT1/2/3 (1:1000), anti-Flk1(A3) (1:1000), anti-ubiquitin P4D1 (1:1000) from Santa Cruz, anti-p-p44/42 MAPK (1:1000), anti-p-44/42 MAPK (1:1000) from Cell Signaling and Goat Anti-Rabbit IgG-HRP conjugate (1:2000), Goat Anti-mouse IgG-HRP conjugate (1:2000) from Bio-Rad. Protein expression was visualized by ECL (Amersham). Band density was quantified by ImageJ.

Transfections. Control siRNA and ATP7A siRNA were obtained from Applied Biosystems and Sigma Aldrich. Sequence of ATP7A siRNA was as follows: sense, 5'-CUGUAUUAGUAGCAGUUGA-3'; antisense, 5'-UUCUGAUAAAGAUCGG CUGCA-3'. HUVECs were seeded into culture dishes 1 day before transfection. Transfection of siRNA ( $30 \mathrm{nM}$ ) was performed using Oligofectamine (Invitrogen) according to the manufacturer's protocol. VEGF stimulation was performed $48 \mathrm{~h}$ after transfection. For double transfection of siRNA and plasmid DNA, cells were transfected with siRNAs $(20 \mathrm{nM})$ and plasmid DNA ( $3 \mu \mathrm{g}$ for $100 \mathrm{~mm}$ dishes) using JetPRIME (Polyplus, USA). For mRFP-GFP-LC3 plasmid which was a gift from Tamotsu Yoshimori (Addgene plasmid \# 21074; http://n2t.net/ addgene:21074; RRID: Addgene_21074), cells were transfected with DNA (1-3 $\mu \mathrm{g})$ using polyethylenimine (PEI, Polysciences, USA). After transfection, cells were 
a $\frac{\text { IP: VEGFR2 }}{\text { siCont SIATP7A }}$

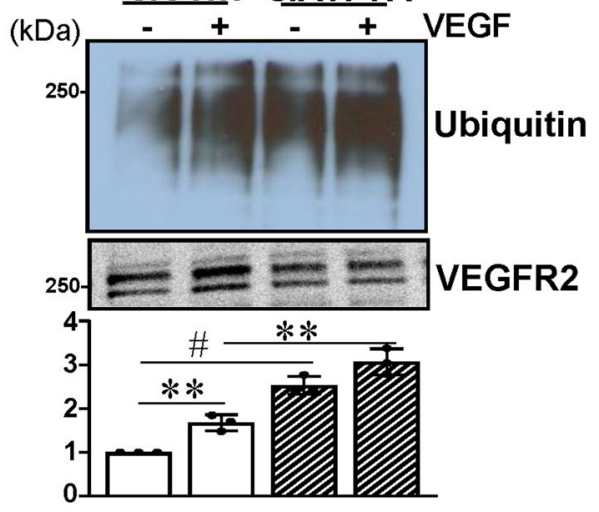

C
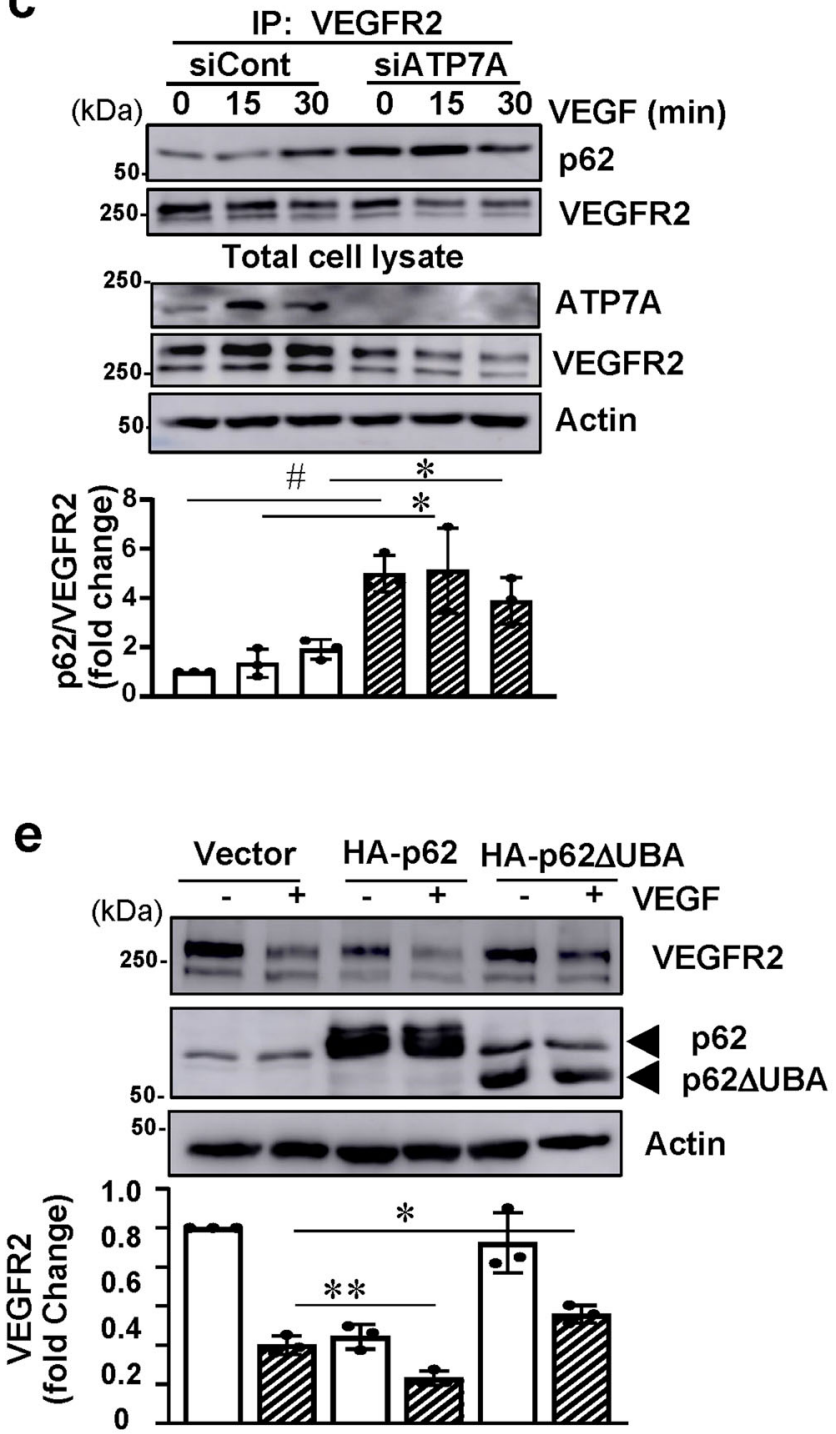
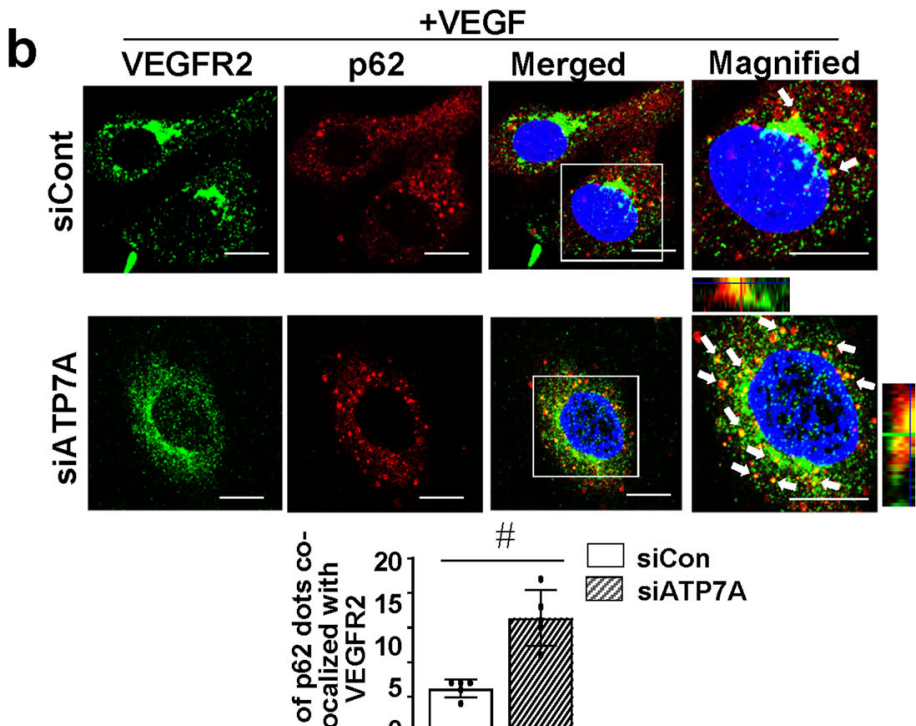
$\stackrel{2}{2}$
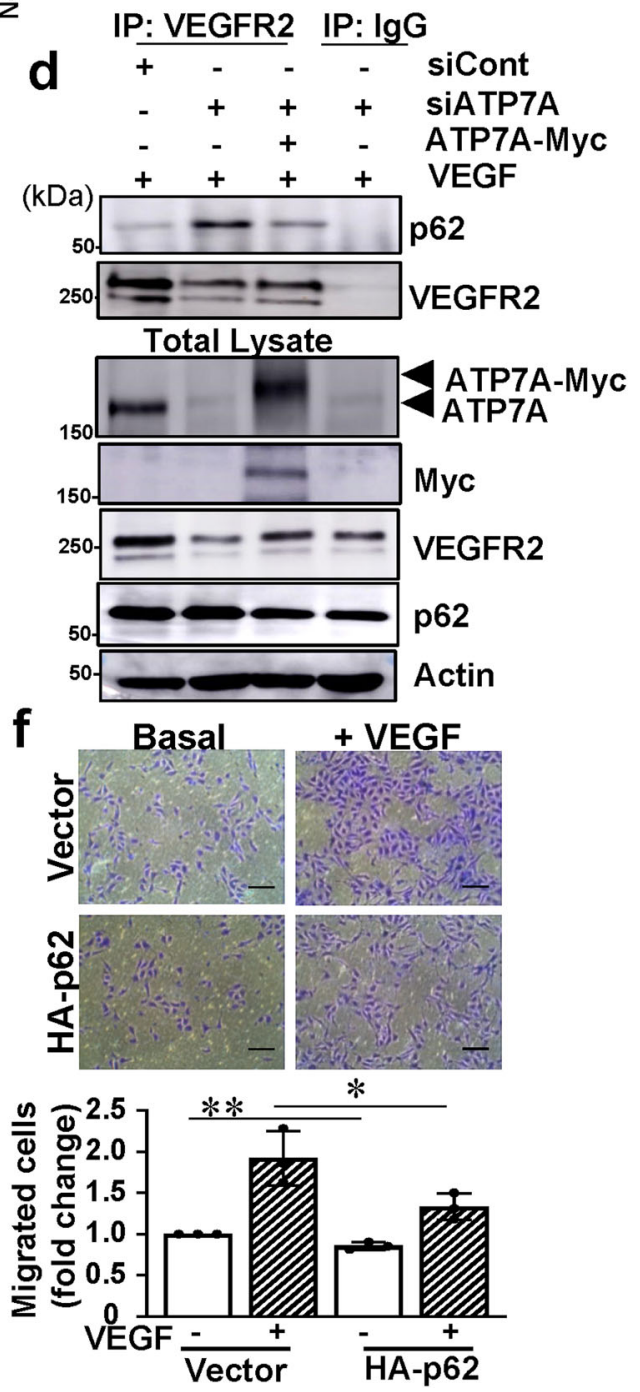

changed to a growth medium and further incubated for $48 \mathrm{~h}$ at $37^{\circ} \mathrm{C}$ before experiments.

Transmission electron microscopy (TEM). HUVECs transfected with siCont or siATP7A were washed with Sorenson buffer ( $\mathrm{pH} 7.4)$ and fixed with $2 \%$ glutaraldehyde in Sorenson buffer ( $\mathrm{pH} 7.4$ ) for $30 \mathrm{~min}$ on $4^{\circ} \mathrm{C}$. The images were taken by
JEM-1220 (JEOL) at the electron microscopy core facility at the University of Illinois at Chicago.

Modified Boyden chamber assay. HUVEC were plated at $1 \times 10^{5}$ cell suspension/ chamber in low serum media with $10 \mathrm{mM}$ dimedone or the vehicle onto the upper chamber of Transwell inserts containing $8 \mu \mathrm{m}$ pore (BD-Biosciences). Chemotaxis 
Fig. 6 ATP7A protects against VEGFR2 degradation by preventing VEGFR2 ubiquitination and its binding with p62. a Human umbilical vein endothelial cells (HUVECs) transfected with control or ATP7A siRNAs were stimulated with vascular endothelial growth factor (VEGF) (20 ng/ml) for $30 \mathrm{~min}$ in the presence of $\mathrm{N}$-ethylmaleimide ( $5 \mathrm{mM}$ ). Lysates were IP with VEGFR2 followed by immunoblotting (IB) with anti Ubiquitin antibody (Ab) to detect VEGFR2 ubiquitination. The bar graph represents averaged fold change over the control. $n=3,{ }^{\star \star} p=0.0031,{ }^{*} p=0.0002$, ${ }^{\star \star} p=0.0023$ (two-tailed unpaired $t$ test). b HUVECs transfected with ATP7A or control siRNAs were stimulated with VEGF $(20 \mathrm{ng} / \mathrm{ml})$ for 30 min and analyzed by immunofluorescence using VEGFR2 and p62 Abs. The scale bar $=10 \mu \mathrm{m}$. Bar graph represents averaged number of VEGFR2 and p62 colocalized dots per cell. $n=5,{ }^{*} p=0.0007$ (two-tailed unpaired $t$-test). c HUVECs transfected with control or ATP7A siRNAs were stimulated with VEGF (20 ng/ml) for 15 and 30 min. Lysates were immunoprecipitated (IP) with VEGFR2 Ab followed by IB with p62 or VEGFR2 Abs. $n=3$ independent experiments, ${ }^{*} p=0.0007,{ }^{\star} p=0.0232,{ }^{\star} p=$ 0.0294 (two-tailed unpaired $t$-test). d Bovine aortic endothelial cells (BAEC) transfected with bovine control or ATP7A siRNAs along with empty vector or ATP7A-Myc plasmid and cells were stimulated with VEGF $(20 \mathrm{ng} / \mathrm{ml})$ for $30 \mathrm{~min}$. Lysates were IP with VEGFR2 Ab followed by IB with p62 or VEGFR2 Abs. $(n=3)$. e HUVECs transfected with Adenovirus expressing p62 WT or p62 lacking ubiquitin-associated (UBA) domain were treated with VEGF $\left(20 \mathrm{ng} / \mathrm{ml}\right.$ ) for $60 \mathrm{~min}$. Lysates were used for IB with VEGFR2, HA-p62. $n=3$ independent experiments, ${ }^{\star} p=0.0145,{ }^{\star \star}{ }^{\star} p=0.0082$ (two-tailed unpaired $t$ test). $\mathbf{f}$ HUVECs transfected with empty vector or HA-p62 were used to measure basal and VEGF-induced endothelial cell (EC) migration using the modified Boyden chamber method. Bar graph represents averaged fold change over control. Scale bars $=100 \mu \mathrm{m} . n=3,{ }^{\star \star} p=0.0059,{ }^{\star} p=0.0499$ (twotailed unpaired t-test). Data are mean \pm SEM.

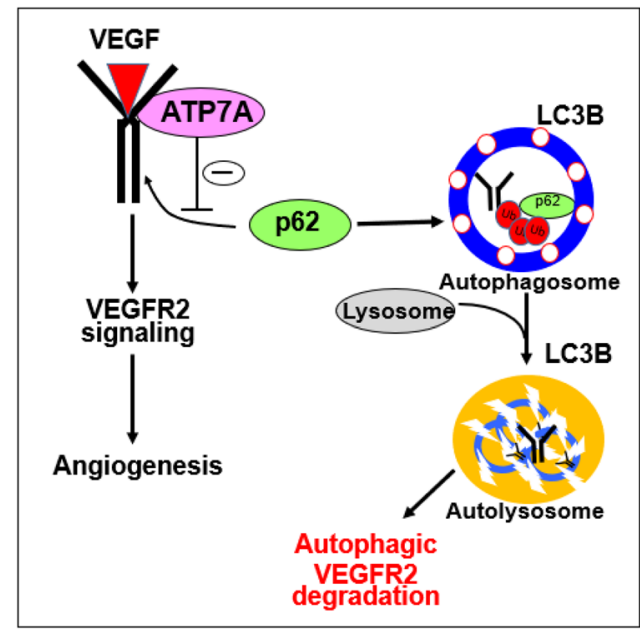

Fig. 7 Proposed model. Proposed model showing that ATP7A binds to VEGFR2 in endothelial cells in response to VEGF, which protects against p62/SQSTM1-mediated autophagic and lysosomal degradation of VEGFR2. This in turn promotes VEGFR2 signaling, thereby promoting angiogenesis and ischemic neovascularization.

was achieved by the presence of $50 \mathrm{ng} / \mathrm{ml}$ VEGF with $10 \mathrm{mM}$ dimedone or vehicle in a $37^{\circ} \mathrm{C}$ culture incubator. After $6 \mathrm{~h}$, transwell chambers were rinsed with PBS, and the cells on top were removed with a cotton tip applicator. Cells on the bottom of the transwell were fixed in 100\% methanol and stained with Diff-Quick Stain kit from Imed Inc. Eight random fields per transwell were imaged with a Nikon digital camera, and the number of cells was counted by using NIH Image J software.

3D spheroid sprouting assay. To generate HUVEC spheroids, the endothelial cells were cultured overnight in a complete medium containing $0.25 \%$ carboxyl methylcellulose (CMC) in non-adherent U bottom 96 well plates. Harvested spheroids were overlaid with 0.8\% CMC and 20\% FBS solution in M199 media and collagen solution added in a 1:1 v/v ratio and plated on pre wormed 24 well plates to form the gel. The gels were overlaid with a complete medium supplemented with $50 \mathrm{ng} / \mathrm{ml}$ VEGF. Twenty-four hours after embedding the cumulative length of the sprouts growing out of each spheroid, their mean number and length were measured using ImageJ software on bright-field images.

Cell surface biotinylation assay. The cell surface VEGFR2 or ATP7A was biotinylated following exposure to VEGF $(20 \mathrm{ng} / \mathrm{ml})$ for the indicated time. Biotinylation of cells was carried out using the cell-impermeable, thiol-cleavable SulfoNHS-SS-biotin (Pierce) reagent to label cell surface proteins. All of the biotinylation procedures were carried out at $4{ }^{\circ} \mathrm{C}$. Briefly, cell-surface proteins were labeled for $45 \mathrm{~min}$ with $0.2 \mathrm{mg} / \mathrm{ml}$ cleavable water-soluble cell-impermeable sulpho-NHSSS-biotin (Pierce Endogen) in PBS supplemented with $0.5 \mathrm{mM} \mathrm{MgCl} 2$ and $1 \mathrm{mM}$ $\mathrm{CaCl}$. The unbound sulfo-NHS-SS-biotin was quenched by $50 \mathrm{mM}$ Tris (pH 8.0) containing $100 \mathrm{mM} \mathrm{NaCl}$. The cells were lysed in $500 \mu \mathrm{l}$ of ice-cold lysis buffer, $\mathrm{pH}$ 7.4 (50 mM HEPES, $5 \mathrm{mM}$ EDTA, $100 \mathrm{mM} \mathrm{NaCl}$ ), $1 \%$ Triton X-100, $60 \mathrm{mM} \mathrm{n}$ Octyl- $\beta$-D-glucopyranoside, protease inhibitors $(10 \mu \mathrm{g} / \mathrm{ml}$ aprotinin, $1 \mathrm{mM}$ phenylmethylsulfonyl fluoride, $10 \mu \mathrm{g} / \mathrm{ml}$ leupeptin) and phosphatase inhibitors (50 mM sodium fluoride, $1 \mathrm{mM}$ sodium orthovanadate, $10 \mathrm{mM}$ sodium pyrophosphate), and precipitated on streptavidin-agarose beads (Pierce Endogen) overnight. The beads were washed three times in lysis buffer and were eluted in loading buffer. Proteins were subjected to Western immunoblot analysis to detect biotinylated VEGFR2 or ATP7A.

Isolation of mouse aortic endothelial cells (MAECs). Primary MAECs were isolated as follows ${ }^{77}$. Mice were anesthetized and perfused with PBS containing $1000 \mathrm{U} / \mathrm{mL}$ of heparin. The aorta was removed from the mouse and washed with ice-cold PBS, and the surrounding fat tissues were removed. The aorta was then immediately transferred to endothelial growth medium and cut into $2 \mathrm{~mm}$ small segments. The collagen gel was prepared by diluting type I collagen with an endothelial growth medium. The collagen gel was added to 6-well plates $(1.5 \mathrm{ml}$ per well) and allowed to solidify at $37^{\circ} \mathrm{C}$ for at least $30 \mathrm{~min}$. The aorta was cut open and carefully laid on a collagen gel with the endothelium directly facing the gel. The aorta explants were incubated at $37^{\circ} \mathrm{C}$ in an endothelial growth medium. The aorta fragments were removed after 2 days. The remaining adherent endothelial cells were allowed to grow on the matrix for additional 3 days. Thereafter, the cells were harvested by $0.25 \%$ Trypsin solution and centrifuged for $5 \mathrm{~min}$, and placed onto a $0.1 \%$ gelatin-coated flask.

Proximity ligation assay (PLA). An in situ proximity ligation assay (PLA) was performed by using Duolink In Situ PLA reagents (Sigma-Aldrich) to assess the endogenous interaction of ATP7A and VEGFR2 with high specificity and sensitivity. Cells were fixed with $4 \%$ paraformaldehyde for $10 \mathrm{~min}$, and then permeabilized in $0.05 \%$ Triton X-100 in PBS for $5 \mathrm{~min}$ and incubated with primary antibodies against ATP7A (mouse monoclonal, 1:200, Life Span Biosciences, Cat\# LS-B8162)) and VEGFR2 (rabbit monoclonal, 1:200, Cell Signaling, Cat\# 2479 S) for overnight at $4{ }^{\circ} \mathrm{C}$. After washing, cells were treated with secondary anti-mouse and anti-rabbit antibodies conjugated with oligonucleotides of a PLA probe and then subjected to oligonucleotide hybridization, ligation, amplification, and detection following the manufacturer's instructions. In this assay, a positive signal is created only when the epitopes of the target proteins are in close proximity $(<40$ $\mathrm{nm}$ ). Finally, the signal from each detected pair of PLA probes in the cells with a mounting medium containing DAPI was then counted under Zeiss Confocal LSM 510 microscope $\left(\lambda_{\text {ex }} 594 \mathrm{~nm} ; \lambda_{\text {em }} 624 \mathrm{~nm}\right)$. For negative control, cells were treated with single or no primary antibody.

LOX activity assay. LOX activity from cell lysate was measured using a highsensitivity fluorescence assay ${ }^{78}$. Cells were homogenized in $1 \times$ LOX Urea buffer and protein estimation was done using a standard method. An equal amount of protein samples was incubated in the presence and absence of $500 \mu \mathrm{M}$ TTM at $37^{\circ}$ C for 30 min with a final reaction mixture supplied by Amplite Fluorimetric Lysyl Oxidase Assay kit (AAT Bioquest) to the manufacturer's instruction. The reaction was stopped on ice, and differences in fluorescence intensity (540-nm excitation wavelength and 590-nm emission wavelength) between samples with and without TTM were determined. Specific activity was determined by the ratio of activity to the relative amount of protein.

Quantitative real-time PCR. Total RNA was isolated by using Tri Reagent (Molecular Research Center, Inc.) and phenol/chloroform. Reverse transcription was carried out using a high-capacity cDNA reverse transcription kit (Applied Biosystems) with $2 \mu \mathrm{g}$ of total RNA. The PCR was performed according to the manufacturer's protocol using ABI PRISM 7000 Sequence Detection System 26 (Applied Biosystems, CA) and QuantiFast SYBR Green PCR Kit (Qiagen, Valenica, Foster City, CA). Primer sequences for qPCR are listed in 
Supplementary Table 1. Samples were all run in triplicates to reduce variability. The expression of genes was normalized and expressed as fold changes relative to HPRT or $18 \mathrm{~S}$

Statistical analysis. Data are presented as mean \pm SEM. Statistical tests were performed using Prism v4 (GraphPad Software, San Diego, CA). Data were compared between groups of cells and animals by a 2-tailed unpaired Student $t$ test. Two-way ANOVA was applied for multiple comparisons, followed by Bonferroni's multiple comparison analysis. The statistical analysis was based on $n$, indicating the number of independent biological samples or mice as described in detail in the respective figure legends. Values of ${ }^{*} p<0.05,{ }^{*} p<0.01, \# p<0.001$ were considered statistically significant.

Reporting summary. Further information on research design is available in the Nature Research Reporting Summary linked to this article.

\section{Data availability}

Data underlying all figures and supplementary figures, uncropped and unprocessed immunoblot scans, and PCR agarose gel pictures for all figures and supplementary figures, are provided as Source Data. All other relevant data are available from the corresponding authors on request. Source data are provided with this paper.

Received: 13 January 2020; Accepted: 26 April 2021;

Published online: 25 May 2021

\section{References}

1. Carmeliet, P. \& Jain, R. K. Molecular mechanisms and clinical applications of angiogenesis. Nature 473, 298-307 (2011).

2. Simons, M., Gordon, E. \& Claesson-Welsh, L. Mechanisms and regulation of endothelial VEGF receptor signalling. Nat. Rev. Mol. Cell Biol. 17, 611-625 (2016).

3. Koch, S., Tugues, S., Li, X., Gualandi, L. \& Claesson-Welsh, L. Signal transduction by vascular endothelial growth factor receptors. Biochem. J. 437, 169-183 (2011).

4. Rahman, H. N. A. et al. Selective targeting of a novel Epsin-VEGFR2 interaction promotes VEGF-mediated angiogenesis. Circulation Res. 118, 957-969 (2016).

5. Lutsenko, S., Barnes, N. L., Bartee, M. Y. \& Dmitriev, O. Y. Function and regulation of human copper-transporting ATPases. Physiol. Rev. 87, 1011-1046 (2007).

6. Fukai, T., Ushio-Fukai, M. \& Kaplan, J. H. Copper transporters and copper chaperones: roles in cardiovascular physiology and disease. Am. J. Physiol. Cell Physiol. 315, C186-C201 (2018).

7. Shanbhag, V. et al. ATP7A delivers copper to the lysyl oxidase family of enzymes and promotes tumorigenesis and metastasis. Proc. Natl Acad. Sci. USA 116, 6836-6841 (2019).

8. Chen, G. F. et al. Copper transport protein antioxidant-1 promotes inflammatory neovascularization via chaperone and transcription factor function. Sci. Rep. 5, 14780 (2015).

9. Kim, H. W., Lin, A., Guldberg, R. E., Ushio-Fukai, M. \& Fukai, T. Essential role of extracellular SOD in reparative neovascularization induced by hindlimb ischemia. Circulation Res. 101, 409-419 (2007).

10. La Fontaine, S. \& Mercer, J. F. Trafficking of the copper-ATPases, ATP7A and ATP7B: role in copper homeostasis. Arch. Biochem. Biophys. 463, 149-167 (2007).

11. Kaler, S. G. ATP7A-related copper transport diseases-emerging concepts and future trends. Nat. Rev. Neurol. 7, 15-29 (2011).

12. Wang, Y., Zhu, S., Weisman, G. A., Gitlin, J. D. \& Petris, M. J. Conditional knockout of the Menkes disease copper transporter demonstrates its critical role in embryogenesis. PLOS ONE 7, e43039 (2012).

13. La Fontaine, S. et al. Intracellular localization and loss of copper responsiveness of Mnk, the murine homologue of the Menkes protein, in cells from blotchy (Mo blo) and brindled (Mo br) mouse mutants. Hum. Mol. Genet. 8, 1069-1075 (1999).

14. Qin, Z. et al. Role of Menkes ATPase in angiotensin II-induced hypertension: a key modulator for extracellular superoxide dismutase function. Hypertension 52, 945-951 (2008).

15. Starcher, B., Madaras, J. A., Fisk, D., Perry, E. F. \& Hill, C. H. Abnormal cellular copper metabolism in the blotchy mouse. J. Nutr. 108, 1229-1233 (1978).

16. Sudhahar, V. et al. Copper transporter ATP7A protects against endothelial dysfunction in type 1 diabetic mice by regulating extracellular superoxide dismutase. Diabetes 62, 3839-3850 (2013).
17. Sudhahar, V. et al. Akt2 stabilizes ATP7A, a copper transporter for SOD3 (Extracellular Superoxide Dismutase), in vascular smooth muscles: novel mechanism to limit endothelial dysfunction in type 2 diabetes mellitus. Arterioscler. Thromb. Vasc. Biol. 38, 529-541 (2018).

18. Nussenzweig, S. C., Verma, S. \& Finkel, T. The role of autophagy in vascular biology. Circulation Res. 116, 480-488 (2015).

19. Katsuragi, Y., Ichimura, Y. \& Komatsu, M. p62/SQSTM1 functions as a signaling hub and an autophagy adaptor. FEBS J. 282, 4672-4678 (2015).

20. Glick, D., Barth, S. \& Macleod, K. F. Autophagy: cellular and molecular mechanisms. J. Pathol. 221, 3-12 (2010).

21. Du, J. et al. Role of autophagy in angiogenesis in aortic endothelial cells. Am. J. Physiol. Cell Physiol. 302, C383-C391 (2012).

22. Liu, H., Yu, S., Zhang, H. \& Xu, J. Angiogenesis impairment in diabetes: role of methylglyoxal-induced receptor for advanced glycation endproducts, autophagy and vascular endothelial growth factor receptor 2. PLOS ONE 7, e46720 (2012)

23. Polishchuk, E. V. et al. Wilson disease protein ATP7B utilizes lysosomal exocytosis to maintain copper homeostasis. Dev. Cell 29, 686-700 (2014).

24. Polishchuk, E. V. et al. Activation of autophagy, observed in liver tissues from patients with Wilson disease and from ATP7B-deficient animals, protects hepatocytes from copper-induced apoptosis. Gastroenterology 156, 1173-1189 e1175 (2019).

25. Masaldan, S. et al. Copper accumulation in senescent cells: Interplay between copper transporters and impaired autophagy. Redox Biol. 16, 322-331 (2018).

26. Wang, Y. et al. Ephrin-B2 controls VEGF-induced angiogenesis and lymphangiogenesis. Nature 465, 483-486 (2010)

27. Ashino, T. et al. Unexpected role of the copper transporter ATP7A in PDGFinduced vascular smooth muscle cell migration. Circulation Res. 107, 787-799 (2010).

28. Heiss, M. et al. Endothelial cell spheroids as a versatile tool to study angiogenesis in vitro. FASEB J. 29, 3076-3084 (2015).

29. Gelfand, M. V. et al. Neuropilin-1 functions as a VEGFR2 co-receptor to guide developmental angiogenesis independent of ligand binding. Elife 3, e03720 (2014).

30. Pan, Q. et al. Copper deficiency induced by tetrathiomolybdate suppresses tumor growth and angiogenesis. Cancer Res. 62, 4854-4859 (2002).

31. Brewer, G. J. Copper control as an antiangiogenic anticancer therapy: lessons from treating Wilson's disease. Exp. Biol. Med. 226, 665-673 (2001).

32. Brewer, G. J. Tetrathiomolybdate anticopper therapy for Wilson's disease inhibits angiogenesis, fibrosis and inflammation. J. Cell. Mol. Med. 7, 11-20 (2003).

33. Brewer, G. J. Anticopper therapy against cancer and diseases of inflammation and fibrosis. Drug Discov. 10, 1103-1109 (2005).

34. Medici, V. \& Sturniolo, G. C. Tetrathiomolybdate, a copper chelator for the treatment of Wilson disease, pulmonary fibrosis and other indications. IDrugs 11, 592-606 (2008).

35. Barone, S. et al. Chromosome missegregation in single human oocytes is related to the age and gene expression profile. Int. J. Mol. Sci. 21, 1934 (2020).

36. Turski, M. L. et al. A novel role for copper in Ras/mitogen-activated protein kinase signaling. Mol. Cell Biol. 32, 1284-1295 (2012).

37. Brady, D. C. et al. Copper is required for oncogenic BRAF signalling and tumorigenesis. Nature 509, 492-496 (2014).

38. Söderberg, O. et al. Direct observation of individual endogenous protein complexes in situ by proximity ligation. Nat. Methods 3, 995-1000 (2006)

39. Yamamoto, A. et al. Bafilomycin A1 prevents maturation of autophagic vacuoles by inhibiting fusion between autophagosomes and lysosomes in rat hepatoma cell line, H-4-II-E cells. Cell Struct. Funct. 23, 33-42 (1998).

40. Diaz-Troya, S., Perez-Perez, M. E., Florencio, F. J. \& Crespo, J. L. The role of TOR in autophagy regulation from yeast to plants and mammals. Autophagy 4, 851-865 (2008).

41. Carames, B. et al. Autophagy activation by rapamycin reduces severity of experimental osteoarthritis. Ann. Rheum. Dis. 71, 575-581 (2012).

42. Tanemura, M. et al. Rapamycin causes upregulation of autophagy and impairs islets function both in vitro and in vivo. Am. J. Transpl. 12, 102-114 (2012).

43. Kimura, S., Noda, T. \& Yoshimori, T. Dissection of the autophagosome maturation process by a novel reporter protein, tandem fluorescent-tagged LC3. Autophagy 3, 452-460 (2007).

44. Li, L., Wang, Z. V., Hill, J. A. \& Lin, F. New autophagy reporter mice reveal dynamics of proximal tubular autophagy. J. Am. Soc. Nephrol. 25, 305-315 (2014).

45. Ewan, L. C. et al. Intrinsic tyrosine kinase activity is required for vascular endothelial growth factor receptor 2 ubiquitination, sorting and degradation in endothelial cells. Traffic 7, 1270-1282 (2006).

46. Lee, Y. \& Weihl, C. C. Regulation of SQSTM1/p62 via UBA domain ubiquitination and its role in disease. Autophagy 13, 1615-1616 (2017).

47. Mukherjee, S., Tessema, M. \& Wandinger-Ness, A. Vesicular trafficking of tyrosine kinase receptors and associated proteins in the regulation of signaling and vascular function. Circulation Res. 98, 743-756 (2006). 
48. Petris, M. J. et al. Ligand-regulated transport of the Menkes copper P-type ATPase efflux pump from the Golgi apparatus to the plasma membrane: a novel mechanism of regulated trafficking. EMBO J. 15, 6084-6095 (1996).

49. Simons, M. An inside view: VEGF receptor trafficking and signaling. Physiology 27, 213-222 (2012)

50. Schlief, M. L., Craig, A. M. \& Gitlin, J. D. NMDA receptor activation mediates copper homeostasis in hippocampal neurons. J. Neurosci. 25, 239-246 (2005).

51. White, C. et al. Copper transport into the secretory pathway is regulated by oxygen in macrophages. J. Cell Sci. 122, 1315-1321 (2009).

52. Qin, Z., Itoh, S., Jeney, V., Ushio-Fukai, M. \& Fukai, T. Essential role for the Menkes ATPase in activation of extracellular superoxide dismutase: implication for vascular oxidative stress. FASEB J. 20, 334-336 (2006).

53. Sawamiphak, S. et al. Ephrin-B2 regulates VEGFR2 function in developmental and tumour angiogenesis. Nature 465, 487-491 (2010).

54. Pan, Q. et al. Blocking neuropilin-1 function has an additive effect with antiVEGF to inhibit tumor growth. Cancer Cell 11, 53-67 (2007).

55. van Lessen, M. et al. Regulation of vascular endothelial growth factor receptor function in angiogenesis by numb and numb-like. Arterioscler. Thromb. Vasc. Biol. 35, 1815-1825 (2015).

56. Yamaoka-Tojo, M. et al. IQGAP1, a novel vascular endothelial growth factor receptor binding protein, is involved in reactive oxygen species-dependent endothelial migration and proliferation. Circulation Res. 95, 276-283 (2004).

57. Hung, A. Y. \& Sheng, M. PDZ domains: structural modules for protein complex assembly. J. Biol. Chem. 277, 5699-5702 (2002).

58. Ballmer-Hofer, K., Andersson, A. E., Ratcliffe, L. E. \& Berger, P. Neuropilin-1 promotes VEGFR-2 trafficking through Rabl1 vesicles thereby specifying signal output. Blood 118, 816-826 (2011).

59. Ashino, T. et al. Copper transporter ATP7A interacts with IQGAP1, a Rac1 binding scaffolding protein: role in PDGF-induced VSMC migration and vascular remodeling. Am. J. Physiol. Cell Physiol. 315, C850-C862 (2018)

60. Meyer, R. D. et al. PEST motif serine and tyrosine phosphorylation controls vascular endothelial growth factor receptor 2 stability and downregulation. Mol. Cell Biol. 31, 2010-2025 (2011).

61. Duval, M., Bedard-Goulet, S., Delisle, C. \& Gratton, J. P. Vascular endothelial growth factor-dependent down-regulation of Flk-1/KDR involves Cblmediated ubiquitination. Consequences on nitric oxide production from endothelial cells. J. Biol. Chem. 278, 20091-20097 (2003).

62. Tessneer, K. L. et al. Genetic reduction of vascular endothelial growth factor receptor 2 rescues aberrant angiogenesis caused by epsin deficiency. Arterioscler. Thromb. Vasc. Biol. 34, 331-337 (2014).

63. Pasula, S. et al. Endothelial epsin deficiency decreases tumor growth by enhancing VEGF signaling. J. Clin. Investig. 122, 4424-4438 (2012).

64. Manickam, V. et al. Regulation of vascular endothelial growth factor receptor 2 trafficking and angiogenesis by Golgi localized t-SNARE syntaxin 6. Blood 117, 1425-1435 (2011)

65. Yamada, K. H. et al. KIF13B regulates angiogenesis through Golgi to plasma membrane trafficking of VEGFR2. J. Cell Sci. 127, 4518-4530 (2014).

66. Maghsoudlou, A. et al. RNF121 inhibits angiogenic growth factor signaling by restricting cell surface expression of VEGFR-2. Traffic 17, 289-300 (2016).

67. Warren, C. M., Ziyad, S., Briot, A., Der, A. \& Iruela-Arispe, M. L. A ligandindependent VEGFR2 signaling pathway limits angiogenic responses in diabetes. Sci. Signal. 7, ra1 (2014).

68. Kumar, S. et al. Autophagy triggered by magnolol derivative negatively regulates angiogenesis. Cell Death Dis. 4, e889 (2013).

69. Tsang, T. et al. Copper is an essential regulator of the autophagic kinases ULK1/2 to drive lung adenocarcinoma. Nat. Cell Biol. 22, 412-424 (2020).

70. Sprott, D. et al. Endothelial-specific deficiency of ATG5 (Autophagy Protein 5) attenuates ischemia-related angiogenesis. Arterioscler. Thromb. Vasc. Biol. 39, 1137-1148 (2019).

71. Bjorkoy, G. et al. p62/SQSTM1 forms protein aggregates degraded by autophagy and has a protective effect on huntingtin-induced cell death. J. Cell Biol. 171, 603-614 (2005).

72. Liu, W. J. et al. p62 links the autophagy pathway and the ubiqutin-proteasome system upon ubiquitinated protein degradation. Cell Mol. Biol. Lett. 21, 29 (2016).

73. Igawa, H. et al. p62-mediated autophagy affects nutrition-dependent insulin receptor substrate 1 dynamics in 3T3-L1 preadipocytes. J. Diabetes Investig. 10, 32-42 (2019)
74. Ichimura, Y. et al. Phosphorylation of p62 activates the Keap1-Nrf2 pathway during selective autophagy. Mol. Cell 51, 618-631 (2013).

75. Smirnova, J. et al. Copper(I)-binding properties of de-coppering drugs for the treatment of Wilson disease. alpha-Lipoic acid as a potential anti-copper agent. Sci. Rep. 8, 1463 (2018)

76. Tojo, T. et al. Role of gp91phox (Nox2)-containing NAD(P)H oxidase in angiogenesis in response to hindlimb ischemia. Circulation 111, 2347-2355 (2005).

77. Sudhahar, V. et al. Copper transporter ATP7A (Copper-Transporting P-Type ATPase/Menkes ATPase) limits vascular inflammation and aortic aneurysm development: role of MicroRNA-125b. Arterioscler. Thromb. Vasc. Biol. 39, 2320-2337 (2019)

78. Palamakumbura, A. H. \& Trackman, P. C. A fluorometric assay for detection of lysyl oxidase enzyme activity in biological samples. Anal. Biochem. 300, 245-251 (2002).

\section{Acknowledgements}

This work was supported by NIHR01HL135584 (to M.U.-F.), NIHR01HL133613, NIHR01HL1 16976 (to T.F., M.U.-F.), NIHR01HL070187 (to T.F.), Department of Veterans Affairs Merit Review grant 2I01BX001232 (to T.F.), and 17POST33660754 (to D.A.), Foundation Jerome Lejeune (to M.N.O., J.P.O.), NIHR01HL090651 (to J.P.O.), Department of Defense PR080428 (to J.P.O.), St.Baldrick's Foundation (to J.P.O.), Chicago Biomedical Consortium with support from the Searle Funds at The Chicago Community Trust (to J.P. O.), Center for Clinical and Translational Science at UIC (to J.P.O.), NIH National Center for Advancing Translational Sciences UL1TR00050 (to J.P.O.).

\section{Author contributions}

M.U.-F., T.F., D.A., V.S., S.W.-Y. designed the study; D.A., V.S., S.W.-Y., M.N.O., J.P.O A.D., M.M., Y.H. performed research; M.U.-F., T.F., D.A., V.S., S.W.-Y. analyzed data; J.P.O., J.H.K. discussed data and provided inputs; M.U.-F., T.F., D.A., V.S. wrote the manuscript; M.N.O., J.P.O., J.H.K. edited the manuscript. All the authors reviewed the manuscript.

\section{Competing interests}

The authors declare no competing interests.

\section{Additional information}

Supplementary information The online version contains supplementary material available at https://doi.org/10.1038/s41467-021-23408-1.

Correspondence and requests for materials should be addressed to T.F. or M.U.-F.

Peer review information Nature Communications thanks Michael Simons, Pernilla Wittung-Stafshede and the other, anonymous, reviewer(s) for their contribution to the peer review of this work. Peer reviewer reports are available.

Reprints and permission information is available at http://www.nature.com/reprints

Publisher's note Springer Nature remains neutral with regard to jurisdictional claims in published maps and institutional affiliations.

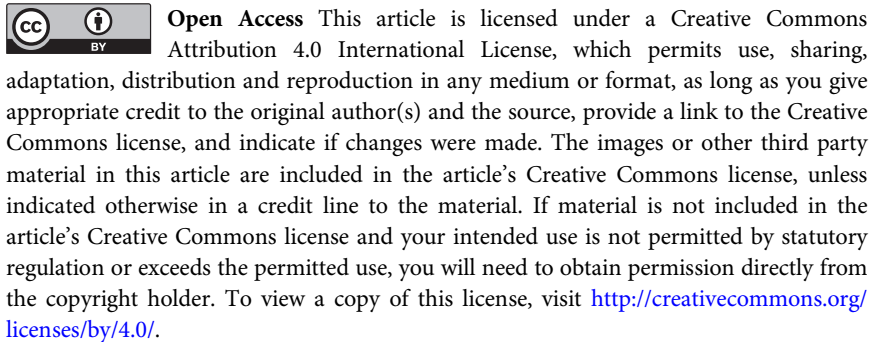

(C) The Author(s) 2021 